\title{
The effect of gravity on liquid plug propagation in a two-dimensional channel
}

\author{
V. Suresh and J. B. Grotberg ${ }^{\text {a) }}$ \\ Department of Biomedical Engineering, University of Michigan, Ann Arbor, Michigan 48109
}

(Received 21 May 2004; accepted 5 October 2004; published online 1 March 2005)

\begin{abstract}
The effect of plug propagation speed and gravity on the quasisteady motion of a liquid plug in a two-dimensional liquid-lined channel oriented at an angle $\alpha$ with respect to gravity is studied. The problem is motivated by the transport of liquid plugs instilled into pulmonary airways in medical treatments such as surfactant replacement therapy, drug delivery, and liquid ventilation. The capillary number $\mathrm{Ca}$ is assumed to be small, while the Bond number $B o$ is arbitrary. Using matched asymptotic expansions and lubrication theory, expressions are obtained for the thickness of the trailing films left behind by the plug and the pressure drop across it as functions of $\mathrm{Ca}, \mathrm{Bo}, \alpha$ and the thickness of the precursor films. When the Bond number is small it is found that the trailing film thickness and the flow contribution to the pressure drop scale as $\mathrm{Ca}^{2 / 3}$ at leading order with coefficients that depend on $B o$ and $\alpha$. The first correction to the film thickness is found to occur at $O(\mathrm{Ca})$ compared to $O\left(\mathrm{Ca}^{4 / 3}\right)$ in the $B o=0$ case. Asymmetry in the liquid distribution is quantified by calculating the ratio of liquid volumes above and below the centerline of the channel, $V_{R} \cdot V_{R}$ $=1$ at $B o=0$, indicating a symmetric distribution, and decreases with $B o$ and $C a$, but increases with the plug length $L_{p}$. The decrease of $V_{R}$ with $C a$ suggests that higher propagation speeds in small airways may result in less homogenous liquid distribution, which is in contrast to the expected effect in large airways. For given values of the other parameters, a maximum capillary number $C a_{\mathrm{c}}$ is identified above which the plug will eventually rupture. When the Bond number becomes equal to an orientation-dependent critical value $B o_{c}$, it is found that the scaling of the film thickness and pressure drop change to $C a^{1 / 2}$ and $C a^{1 / 6}$, respectively. It is shown that this scaling is valid for small increments of the Bond number over its critical value, $B o=B o_{c}+B C a^{1 / 6}$, but for higher Bond numbers the asymptotic approach breaks down. (C) 2005 American Institute of Physics.
\end{abstract}

[DOI: 10.1063/1.1863853]

\section{INTRODUCTION}

Liquid is instilled into the lungs during medical treatments such as surfactant replacement therapy (SRT), ${ }^{1-3}$ partial liquid ventilation (PLV), ${ }^{4-7}$ and pulmonary drug/genetic material delivery. ${ }^{8-13}$ The instilled liquid may form a meniscus in the trachea ${ }^{14}$ and be transported through the airways in the form of air-blown plugs; it may also drain gravitationally through the larger airways and form plugs in the smaller airways. ${ }^{15}$ The effectiveness of the treatment can depend on: (1) delivery to the desired location via liquid deposition in films left behind by propagating plugs and (2) the homogeneous distribution of liquid through the branching airway network as plugs split at airway bifurcations. In turn, these factors are influenced by a number of parameters including liquid and interfacial properties, propagation speed, gravity, and airway orientation. ${ }^{15-17}$ An understanding of the fluid dynamics of plug propagation would therefore be useful in devising strategies to ensure optimal liquid distribution in the lung.

In this paper we focus on the effect of gravitational acceleration $g$ on plug propagation. Gravitational effects can be important since the Bond number $B o=\rho g a^{2} / \sigma$, based on the airway radius $a$, liquid density $\rho$, and surface tension at the

${ }^{a)}$ Author to whom correspondence should be addressed. air-liquid interface $\sigma$, can vary in the range $10^{-4}-10^{2}$ in typical clinical situations. ${ }^{18}$ In addition, airway orientation with respect to gravity must also be considered since the human airway network has a three-dimensional branching structure. ${ }^{19}$ Consider a plug that moves through a liquidlined airway oriented at an angle $\alpha$ to gravity and splits at an airway bifurcation as shown in Fig. 1. Gravity affects the volume of liquid delivered to each downstream branch in two ways. First, less liquid enters the upper branch since work needs to be done against gravity. Second, gravity causes a larger fraction of the plug to be present below the centerline of the parent airway prior to the bifurcation compared to above, and this asymmetry predisposes a larger fraction of the plug volume to enter the lower branch. This unequal splitting is repeated at each subsequent bifurcation, leading to an overall nonhomogeneous liquid distribution in the lung. One of the aims of this work is to quantify the plug's prebifurcation asymmetry in the liquid distribution and determine its dependence on various parameters.

Gravity and airway orientation also affect the thickness of the films deposited by the plug, which ultimately influences liquid distribution. If the films are thick, the plug loses volume more rapidly and may rupture before it reaches the intended delivery site. On the other hand, if plugs persist over an entire cycle of breathing or are created prior to ex- 


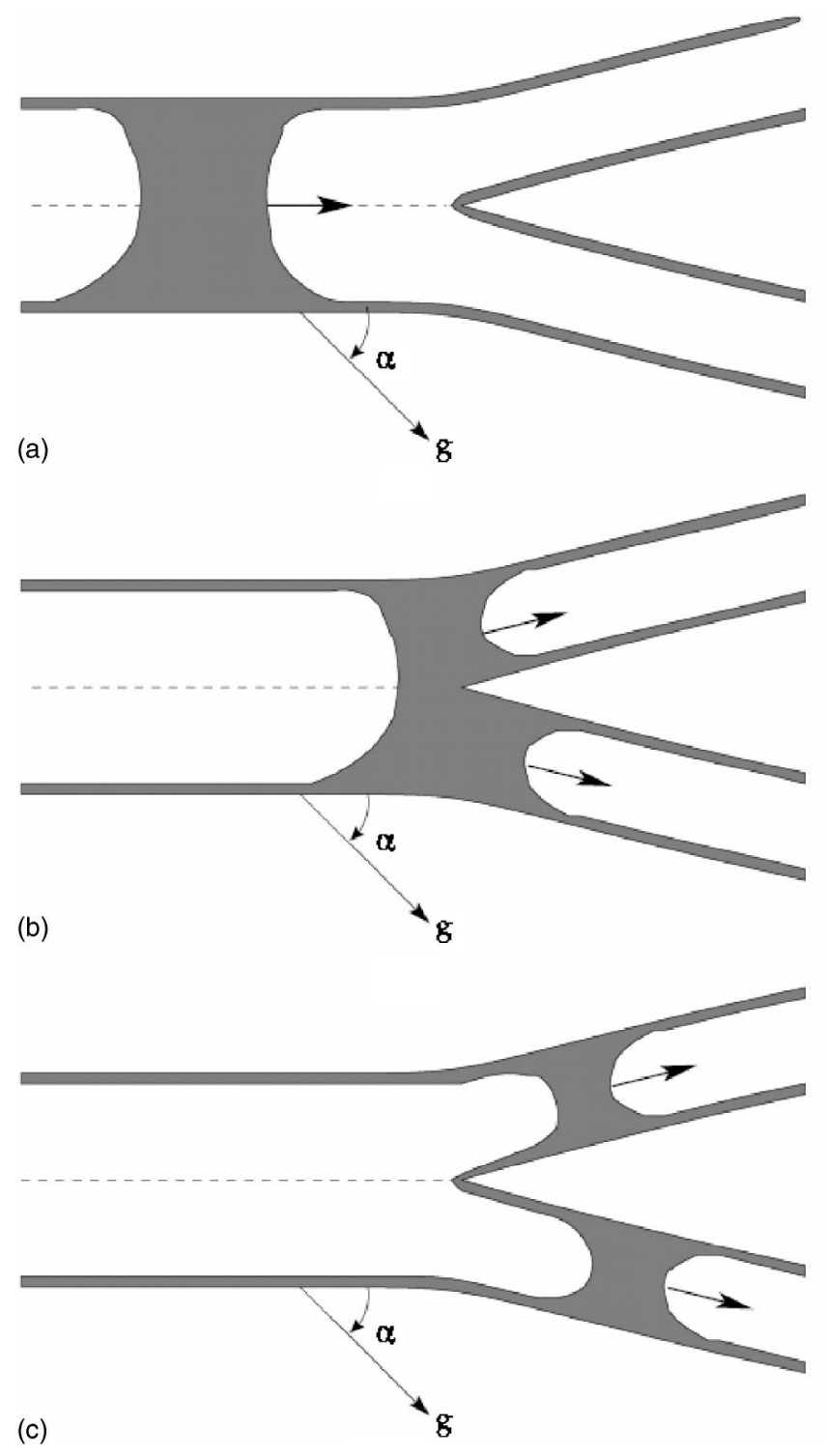

FIG. 1. Schematic of plug motion through an airway bifurcation. (a) A liquid plug moves through a liquid lined airway. The plug volume is asymmetrically distributed about the centerline of the airway due to gravity. (b) The plug enters an airway bifurcation and (c) subsequently splits into two with a larger fraction of the original plug entering the lower, gravity-favored branch.

piration, liquid may be blown out of the lung during exhalation. Therefore, a second aim of this work is to determine how gravity and airway orientation affect the possibility of plug rupture.

In the smaller distal airways, the plug propagation speed $\bar{U}$ is typically slow and the capillary number $C a=\mu \bar{U} / \sigma$, where $\mu$ is the liquid viscosity, is small. The problem of plug propagation in a cylindrical geometry in the presence of gravity is highly complicated due to the lack of azimuthal symmetry. In order to simplify the analysis we study the low capillary number, pressure driven motion of a liquid plug through a two-dimensional channel oriented at an angle $\alpha$ with respect to gravity. This simplification neglects the destabilizing radial component of interfacial curvature that is responsible for a Rayleigh instability of the liquid film that can lead to the formation of liquid plugs in airways. The focus of this work is on preexisting plugs that are either introduced into airways during a medical procedure or form due to instabilities. In particular, the prebifurcation asymmetry and liquid deposition depend on the effect of gravity on the longitudinal curvature of the interface, which is retained in the two-dimensional model.

An analysis of the low capillary number motion of a long bubble through a tube was carried out by Bretherton ${ }^{20}$ who found that the thickness of the liquid film separating the bubble and the tube wall and the pressure drop across the bubble both scaled as $\mathrm{Ca}^{2 / 3}$. His work was formalized in the terminology of matched asymptotic expansions by Park and Homsy. ${ }^{21}$ Other authors have examined flow in polygonal capillaries, ${ }^{22,23}$ surfactant effects ${ }^{24-28}$ and thermocapillary migration. ${ }^{29,30}$ In the context of airway plug propagation, small capillary number asymptotic theory has been used to study problems in airway reopening in rigid ${ }^{31}$ and flexible ${ }^{32,33}$ channels and tubes and surfactant effects on plug propagation. ${ }^{34}$ None of these studies considered the effects of gravity.

Jensen et $a l .{ }^{35}$ examined the effect of gravity on the displacement of liquid by an air finger in a horizontal HeleShaw cell using theory and experiment. For $B o<1$ they found that the film thickness followed the $\mathrm{Ca}^{2 / 3}$ scaling of Bretherton with $B o$ dependent coefficients, while the pressure jump across the air-liquid interface was independent of $B o$. For $B o \geqslant 1$ the lower film was an $O(1)$ quantity independent of $\mathrm{Ca}$. Both the film thickness and pressure jump increased with $B o$. The experimentally observed meniscus shapes were in agreement with the theory. Lasseux and Quintard $^{36}$ and Lasseux ${ }^{37}$ studied gravitational gas-liquid drainage in vertical Hele-Shaw cells and capillary tubes and also found that the Bretherton scaling applies with $B o$ dependent coefficients.

Plug propagation in a tube or channel is closely related to coating flow, a classical example of which is the withdrawal of a fiber or plate from a liquid bath. The review by Quéré ${ }^{38}$ provides an excellent overview of existing literature. Early work found the film thickness to scale as $\mathrm{Ca}^{2 / 3}$ when gravitational drainage in the entrained film was neglected. ${ }^{39,40}$ These results were recast in the form of matched asymptotic expansions by Wilson ${ }^{41}$ and extended to account for nonvertical withdrawal. In the gravity dominated case a thickness proportional to $\mathrm{Ca}^{1 / 2}$ was predicted. ${ }^{42}$ Approximations for the crossover between the two regimes showed reasonable agreement with experiment. ${ }^{43,44}$

The small $\mathrm{Ca}$ asymptotic analysis of gas-liquid displacement and coating flows shows that the same scaling laws arise in both situations: When gravitational effects are small a static meniscus is connected to a thin film of thickness $t$ $\sim \mathrm{Ca}^{2 / 3}$ by a dynamic meniscus of length $l \sim \mathrm{Ca}^{1 / 3}$. When gravitational effects are strong, crossover to a different scaling regime with $t \sim \mathrm{Ca}^{1 / 2}$ occurs in coating flows. In this paper we show that a similar crossover occurs when $B o$ is increased for plug propagation in an inclined channel. The paper is organized as follows. In Sec. II we describe the model and discuss the basic equations and scaling arguments in different regimes. In Secs. III and IV we provide a systematic development of the asymptotic equations and their 


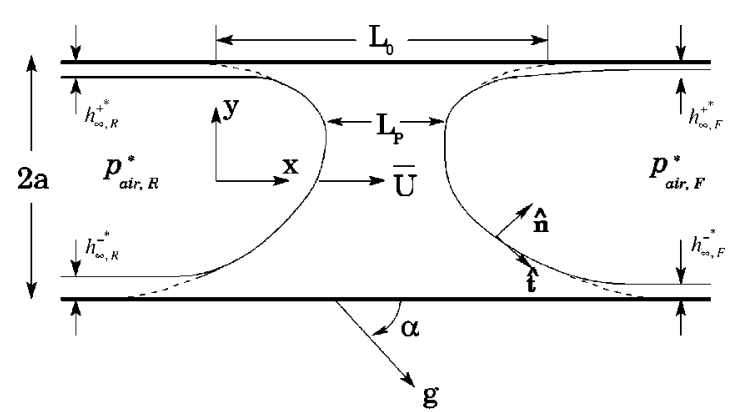

FIG. 2. Schematic of the model showing the propagating plug and thin films. The dashed lines represent the static solutions for the rear and front interfaces, and $x=0$ is located at the intersection of the static rear interface with the upper wall. $L_{0}$ is the distance between the contact points of the rear and front static solutions with the upper wall. $L_{p}$ is the length of the plug and is equal to the distance between the tips of the two interfaces. $L_{0}$ and $L_{p}$ are not independent parameters, see Sec. III A for explanation.

solutions. We conclude in Sec. V with a discussion of the results and their applicability to human airways.

\section{FORMULATION}

\section{A. Model description}

A schematic of the model is shown in Fig. 2. It consists of a plug of incompressible, Newtonian fluid of viscosity $\mu$ and density $\rho$ moving at constant speed $\bar{U}$ in the laboratory frame through a two-dimensional channel of thickness $2 a$. The channel is oriented at an angle $\alpha$ with respect to the gravitational acceleration $g$ and lined with thin precursor films of the plug fluid. The plug separates two semiinfinite air fingers of negligible viscosity and density. The surface tension at the air-liquid interface is assumed to be constant and equal to $\sigma$.

Dimensional variables are denoted by superscript ${ }^{*}$. We use a Cartesian coordinate system $\left(x^{*}, y^{*}\right)$ with the channel walls located at $y^{*}= \pm a$ and seek a steady solution in the frame of the moving plug. Fluid velocity and pressure in the plug fluid are denoted by $\mathbf{u}^{*}=\left(u^{*}, v^{*}\right)$ and $p^{*}$ respectively. Air pressures behind and ahead of the plug are constant and equal to $p_{\text {air } R}^{*}$ and $p_{\text {air }, F}^{*}$. The air-liquid interface is located at $y^{*}=h^{ \pm *}\left(x^{*}\right)$. Superscript ${ }^{+}$and ${ }^{-}$denote quantities in the upper and lower parts of the channel, respectively. As the plug moves through the channel, it deposits trailing films on the upper and lower walls, whose thicknesses $h_{\infty, R}^{+*}$ and $h_{\infty, R}^{-*}$ will be determined. The corresponding precursor film thicknesses ahead of the plug are specified and equal to $h_{\infty, F}^{+*}$ and $h_{\infty, F}^{-*}$. Subscript " $R$ " and " $F$ " denote quantities behind (rear) and ahead (front) of the plug, and will be dropped when an expression applies both ahead and behind the plug.

\section{B. Dimensionless groups and governing equations}

Lengths are scaled by the channel half-width $a$, velocities by the propagation speed $\bar{U}$, and pressures by the capillary scale $\sigma / a$. We assume that the plug motion is suffi-

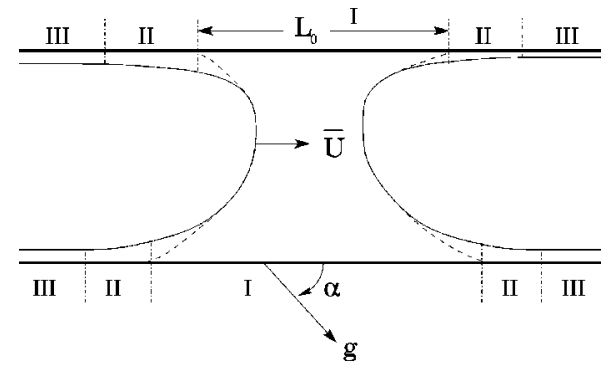

FIG. 3. Schematic showing (I) the statics region close to the body of the plug; (II) four transition regions close to the upper and lower walls at the front and rear meniscus; and (III) trailing and precursor thin films.

ciently slow that inertial effects can be neglected and set the Reynolds number $R e=\rho \bar{U} a / \mu$ equal to zero. Then the relevant dimensionless groups are the capillary number $\mathrm{Ca}$ $=\mu \bar{U} / \sigma$ and the Bond number $B o=\rho g a^{2} / \sigma$. Dropping the superscript ${ }^{*}$, the dimensionless steady Stokes and continuity equations are

$-\nabla p+C a \nabla^{2} \mathbf{u}+B o \hat{\mathbf{g}}=0$,

$\boldsymbol{\nabla} \cdot \mathbf{u}=\mathbf{0}$,

where $\hat{\mathbf{g}}=[\cos (\alpha),-\sin (\alpha)]$ is the unit vector in the direction of gravity. The boundary conditions are

$$
\begin{aligned}
& u=-1, v=0 \text { at } y= \pm 1, \\
& \mathbf{u} \cdot \hat{\mathbf{n}}=0 \text { at } y=h^{ \pm}(x), \\
& \hat{\mathbf{t}} \cdot \boldsymbol{\sigma} \cdot \hat{\mathbf{n}}=0 \text { at } y=h^{ \pm}(x), \\
& p-p_{\text {air }}-\hat{\mathbf{n}} \cdot \boldsymbol{\sigma} \cdot \hat{\mathbf{n}}=\boldsymbol{\nabla} \cdot \hat{\mathbf{n}} \text { at } y=h^{ \pm}(x) .
\end{aligned}
$$

These represent, respectively, no-slip and no penetration at the channel walls, and the kinematic condition, shear, and normal stress balances at the air-liquid interface, $y=h^{ \pm}(x)$. Here $\boldsymbol{\sigma}=C a\left(\boldsymbol{\nabla} \mathbf{u}+\boldsymbol{\nabla} \mathbf{u}^{T}\right)$ is the fluid extra stress tensor, $\hat{\mathbf{n}}$ is the outward pointing unit normal, $\hat{\mathbf{t}}$ is the tangent to the interface, and $\boldsymbol{\nabla} \cdot \hat{\mathbf{n}}$ is the interfacial curvature.

\section{Asymptotic scaling and regimes}

We seek an asymptotic solution of the governing equations in the limit $\mathrm{Ca} \rightarrow 0$. The analysis closely follows that of previous works. ${ }^{21,32,34,35}$ For small $\mathrm{Ca}$ the flow domain can be divided into three regions as shown in Fig. 3: (I) a statics region close to the body of the plug in which surface tension and gravitational body forces are important, but viscous forces are negligible; (II) thin transition regions close to the walls in which viscous, surface tension, and gravitational body forces are important; and (III) flat thin films away from the plug.

Two different force balances are possible in the transition regions, which lead to different scalings for the film thickness as explained below. Suppose that the transition region is a thin layer of length $l$ and thickness $t$, such that $t$ 
$\ll a$. Then the interfacial curvature in the transition region is $\kappa_{t r} \sim t / l^{2}$ leading to a surface tension pressure scale

$$
p_{c a p} \sim \sigma \kappa_{t r} \sim \sigma t / l^{2} .
$$

The viscous pressure scale in the transition region is

$$
p_{v i s} \sim \mu U l / t^{2} \text {. }
$$

First consider the case $g=0$ In the absence of gravity, the viscous and surface tension pressure scales must balance and setting $p_{c a p}=p_{v i s}$ leads to

$$
t / l \sim C a^{1 / 3} .
$$

$\kappa_{t r}$ must match the curvature of the meniscus in the statics region, $\kappa_{s t} \sim 1 / a$, when these regions overlap and so equating the two curvatures results in

$$
t / l^{2} \sim 1 / a .
$$

Equations (7)-(10) lead to the scalings ${ }^{20,21,32,34}$

$$
\begin{aligned}
& l / a \sim C a^{1 / 3}, \\
& t / a \sim C a^{2 / 3}, \\
& p_{t r} /(\sigma / a) \sim 1,
\end{aligned}
$$

where $p_{t r} \sim p_{v i s} \sim p_{s t}$ is the pressure scale in the transition region. We call this the viscocapillary regime based on the dominant forces in the transition region.

When $g \neq 0$ there is also a hydrostatic pressure scale based on the length of the transition region,

$$
p_{g}=\rho g l \cos (\alpha),
$$

and $\kappa_{s t}$ is affected by gravity, i.e., $\kappa_{s t}=\kappa_{s t}(B o, \alpha)$. However, the viscocapillary scaling (11) is valid if the following two conditions are satisfied: (i) the dominant balance in the transition region is between the viscous and surface tension terms [Eq. (9) is valid] and gravitational effects are small compared to these terms, i.e.,

$$
\frac{p_{g}}{p_{\text {vis }}} \sim \frac{p_{g}}{p_{\text {cap }}} \sim C a^{1 / 3} \text { Bo } \cos (\alpha) \ll 1,
$$

and (ii) $\kappa_{s t}(B o, \alpha)$ remains $O(1 / a)$, i.e., (10) holds. These conditions determine constraints on $B o$ and $\alpha$ for which the asymptotic analysis is valid and we will show later that the restriction on $\kappa_{s t}(B o, \alpha)$ is more critical. For $\alpha=\pi / 2$ Jensen et $a l .{ }^{35}$ found that the conditions are satisfied when $B o<1$, $C a \ll 1$.

When $p_{g}$ is the same size as $p_{v i s}$ and $p_{\text {cap }}$ and Bo $\cos (\alpha)=O(1)$ a different scaling is obtained by equating (7), (8), and (12),

$$
\begin{aligned}
& l / a \sim C a^{1 / 6}, \\
& t / a \sim C a^{1 / 2}, \\
& p_{t r} /(\sigma / a) \sim C a^{1 / 6} .
\end{aligned}
$$

We call this the gravitational regime to emphasize the role of gravity in determining the scaling. In this case the curvature in the overlap region is small, $\kappa_{s t} \sim t / l^{2} \sim \mathrm{Ca}^{1 / 6} / a \ll 1 / a$. Also the film thickness $t$ and transition region length $l$ are larger and $p_{t r}$ smaller by $O\left(\mathrm{Ca}^{1 / 6}\right)$ compared to the viscocapillary scaling, (11). We will show in the following sections that the viscocapillary scaling holds for small $B o$ and that for each orientation $\alpha$, there is a critical $B o$ at which the scaling changes to (14). Note that $t \ll l$ for both scalings, and so the hydrostatic variation of pressure over the depth of the transition region can be neglected.

\section{Perturbation expansion}

Velocities, pressures, interface position, and film thickness are written in terms of a perturbation expansion for small $\mathrm{Ca}$. From the preceding analysis a natural choice for the expansion parameter is $\mathrm{Ca}^{a}$ with $a=1 / 3$ for the viscocapillary regime and $a=1 / 6$ for the gravitational regime. Thus

$$
f=f_{0}+C a^{a} f_{1}+C a^{2 a} f_{2}+\cdots,
$$

where $f$ can be $u, v, p, h$, or $t$. In the transition regions, rescaled variables are defined as

$$
\begin{aligned}
& X=\frac{x-x_{0}^{ \pm}}{C a^{a}}, Y=\frac{1 \mp y}{C a^{b}}, H^{ \pm}=\frac{1 \mp h^{ \pm}}{C a^{b}}, T^{ \pm}=\frac{h_{\infty}^{ \pm}}{C a^{b}}, \\
& (U, V)=\left(u, \frac{v}{C a^{b-a}}\right), P=\frac{p}{C a^{c}},
\end{aligned}
$$

where $b=2 / 3, c=0$ for the viscocapillary regime and $b$ $=1 / 2, c=1 / 6$ for the gravitational regime. The upper (lower) sign in \pm , $\mp$ refers to the upper (lower) transition regions. The origins of the transition regions are located at $\left(x_{0}^{ \pm}, \pm 1\right)$ and the interface is located at $Y=H^{ \pm}(X)$. The velocity scaling follows from the continuity equation (2) and the no-slip boundary condition (3). $U, V, P, H^{ \pm}$, and $T^{ \pm}$are also expanded in a perturbation series of the form (15).

\section{E. Matching conditions}

The transition regions must be matched to the statics region and the thin films at either end. The matching to the thin films requires that the transition region thickness equal the film thickness far from the plug, i.e., $H^{ \pm}(X) \rightarrow T^{ \pm}, X$ $\rightarrow \mp \infty$. The limits $-\infty$ and $+\infty$ are valid in the rear and front transition regions, respectively. At the other end, the transition regions overlap with the statics region as $X \rightarrow \pm \infty$ and $x \rightarrow x_{0}^{ \pm}$. Therefore, the matching criterion is obtained from (16) to be

$$
\lim _{x \rightarrow x_{0}^{ \pm}} h^{ \pm}(x)=\lim _{X \rightarrow \begin{cases}+\infty & \text { (rear) } \\ -\infty & \text { (front) }\end{cases} } \pm\left(1-C a^{b} H^{ \pm}(X)\right) .
$$

As described by Park and Homsy, ${ }^{21}$ matching conditions at each order are found by expanding $h^{ \pm}(x)$ in a Taylor series about $x=x_{0}^{ \pm}$, rewriting in terms of the transition region variables and comparing term by term with the right-hand side of (17). 


\section{THE VISCOCAPILLARY REGIME}

\section{A. $O\left(\mathrm{Ca}^{0}\right)$ equations in the statics region}

Viscous terms are negligible in (1) up to $O(C a)$, and the pressure is hydrostatic at leading order,

$$
p_{0}(x, y)=\text { Box } \cos (\alpha)-\text { Boy } \sin (\alpha)+c_{0} .
$$

The normal stress boundary condition (6) takes the form

$$
\text { Box } \cos (\alpha)-B o h_{0}^{ \pm}(x) \sin (\alpha)-\delta p_{0}= \pm \frac{h_{0, x x}^{ \pm}}{\left(1+h_{0, x}^{ \pm}\right)^{3 / 2}},
$$

where $\delta p_{0}=\delta p_{0, R}=p_{a i r, R}-c_{0}$ for the rear meniscus and $\delta p_{0}$ $=\delta p_{0, F}=p_{\text {air }, F}-c_{0}$ for the front meniscus. Thus (19) provides differential equations to determine the shape of the two menisci. A Taylor series expansion of $h_{0}^{ \pm}(x)$ up to $O\left(\mathrm{Ca}^{1 / 3}\right)$ in (17) gives the boundary conditions

$$
\begin{aligned}
& h_{0}^{ \pm}\left(x_{0}^{ \pm}\right)= \pm 1, \\
& h_{0, x}^{ \pm}\left(x_{0}^{ \pm}\right)=0 .
\end{aligned}
$$

In addition, $h_{0}^{+}(x)$ and $h_{0}^{-}(x)$ must join smoothly at the "tip" of the meniscus, $x=x_{t}$, where their derivatives are unbounded, i.e.,

$$
h_{0}^{+}=h_{0}^{-}, \quad h_{0, x}^{ \pm} \rightarrow \mp \infty x=x_{t} .
$$

Equation (22) applies at all orders in $\mathrm{Ca}$. For the rear interface, the origin of the coordinate system is specified by choosing $x_{0, R}^{+}=0$. Specifying $L_{0}$ then fixes $x_{0, F}^{+}$for the front interface (see Fig. 2). For each interface, the four constraints (20) and (21) along with the smoothness condition (22) specify boundary conditions for (19) and determine $\delta p_{0}$ and $x_{0}^{-}$. Equations (20) and (21) state that the static solution at $O\left(\mathrm{Ca}^{0}\right)$ apparently meets the channel walls with a zero contact angle. For any given $L_{0}$, the plug length $L_{P}$ can be determined after solving for the static interfaces. The difference in air pressures between the rear and front of the plug $\Delta p_{0}$ $=p_{\text {air }, R}-p_{\text {air }, F}=\delta p_{0, R}-\delta p_{0, F}$ depends on $L_{0}$ (equivalently, the plug length $L_{P}$ ) and counteracts the gravitational body force acting on the plug, preventing it from accelerating.

It can be shown that the front interface satisfies the same equations and boundary conditions as the rear interface when $\left(x, \alpha, \delta p_{0, F}\right)$ are replaced by $\left[L_{0}-x, \pi-\alpha, \delta p_{0, R}+L_{0} \cos (\alpha)\right]$. Therefore, if the solution for the rear interface $y=h_{0, R}^{ \pm}(x)$ and the pressure jump $\delta p_{0, R}$ are known for some $(B o, \alpha)$ and $L_{0}$ is specified, the solution for the front interface $y=h_{0, F}^{ \pm}(x)$ and the pressure jump $\delta p_{0, F}$ are known for $(B o, \pi-\alpha)$. In contrast to the $B o=0$ case, (19)-(21) do not admit an analytical solution. The system is transformed to arc-length coordinates as described in the Appendix and integrated numerically using the boundary value solver COLMOD which is available for download from the internet at http://www.netlib.org. ${ }^{45,46}$

\section{B. $O\left(\mathrm{Ca}^{0}\right)$ equations in the transition regions}

To derive the transition region equations, the rescaled variables (16) and the perturbation expansion (15) are substituted into (1)-(6). The momentum and continuity equations reduce to the lubrication equations. Using the lubrication velocity field in the kinematic and normal stress conditions results in a third-order ordinary differential equation for the interface location. The details of the derivation have been discussed by previous authors, ${ }^{20,21}$ and carry through with minor modifications when gravity is included. Therefore, we do not repeat the details here and only present the final result that the interface shape is given by the Landau-Levich equation

$$
J_{0, \xi \xi \xi}^{ \pm}=\frac{3\left(J_{0}^{ \pm}-1\right)}{\left(J_{0}^{ \pm}\right)^{3}},
$$

where

$$
J_{0}^{ \pm}=H_{0}^{ \pm} / T_{0}^{ \pm}, \quad \xi=\left(X+X_{0}^{ \pm}\right) / T_{0}^{ \pm} .
$$

Here $X_{0}^{ \pm}$is an arbitrary translation of the coordinates, which will be determined by matching to the statics region. Appropriate initial conditions for integrating (23) are obtained from the asymptotic behavior of $J_{0}^{ \pm}$in the limit $\xi \rightarrow-\infty$ in the rear and $\xi \rightarrow+\infty$ in the front, where the interface must match the constant film thickness. The initial conditions are of the form

$$
\begin{aligned}
& J_{0}^{ \pm} \sim 1+\exp (\lambda \xi), \quad \xi \rightarrow-\infty(\text { rear }), \\
& J_{0}^{ \pm} \sim 1+A \exp (-\lambda \xi / 2) \cos (\sqrt{3} \lambda \xi / 2), \quad \xi \rightarrow+\infty \text { (front) },
\end{aligned}
$$

where $\lambda=3^{1 / 3}$ and $A$ is a free parameter that depends on the precursor film thickness as explained below.

Numerical integration of (23) shows that the asymptotic form of $J_{0}^{ \pm}$close to the statics region is

$$
J_{0}^{ \pm} \sim\left(A_{0}^{ \pm} / 2\right) \xi^{2}+A_{1}^{ \pm} \xi+A_{2}^{ \pm}, \quad \xi \rightarrow \pm \infty .
$$

Applying the matching condition (17) at $O\left(\mathrm{Ca}^{2 / 3}\right)$ the film thickness $T_{0}^{+}$and $T_{0}^{-}$on the upper and lower walls, respectively, are found to be

$$
T_{0}^{ \pm}=\frac{A_{0}^{ \pm}}{h_{0, x x}^{ \pm}\left(x_{0}^{ \pm}\right)} .
$$

For the trailing films the integration gives $A_{0}^{ \pm}=1.3375$. Note that $\pm h_{0, x x}^{ \pm}\left(x_{0}^{ \pm}\right)$is the curvature at $x_{0}^{ \pm}$since $h_{0, x}^{ \pm}\left(x_{0}^{ \pm}\right)=0$, and thus the film thickness is inversely proportional to the local curvature of the statics solution. When $B o=0$, it is easy to show that $h_{0, x x}^{ \pm}\left(x_{0}^{ \pm}\right)=\mp 1$ and (27) reduces to the wellknown result of Bretherton. ${ }^{20}$ Results for $B o \geqslant 0$ and different $\alpha$ are plotted in Figs. 4(a) and 4(b). On the lower wall, $T_{0, R}$ decreases with increasing $B o$ for $\alpha=0$ and passes through a slight maximum for $\alpha=\pi / 4$. For $\alpha \geqslant \pi / 2, T_{0, R}^{-}$ increases monotonically with $B o$ and diverges at a finite critical value $B o=B o_{\mathrm{c}}$ when $h_{0, x x}^{-}\left(x_{0}^{-}\right)$becomes equal to zero. The upper film is thinner than the lower film $\left(T_{0, R}^{+}<T_{0, R}^{-}\right)$, except for $\alpha=0$ and $\pi$ when $T_{0, R}^{+}=T_{0, R}$ due to symmetry. $T_{0, R}^{+}$, in general, decreases with $B o$ and does not diverge except for $\alpha=\pi$. These results can be summarized as follows: For $\alpha$ $<\pi / 2$, viscocapillary solutions can be found for the rear transition regions in which the film thickness on either wall is finite. For $\alpha \geqslant \pi / 2$, viscocapillary solutions only exist for Bond numbers less than a critical value $B o_{\mathrm{c}}$ at which the film thickness on the upper/lower walls diverges. This critical value marks the transition to the gravitational regime, which is discussed in greater detail in Sec. IV. 

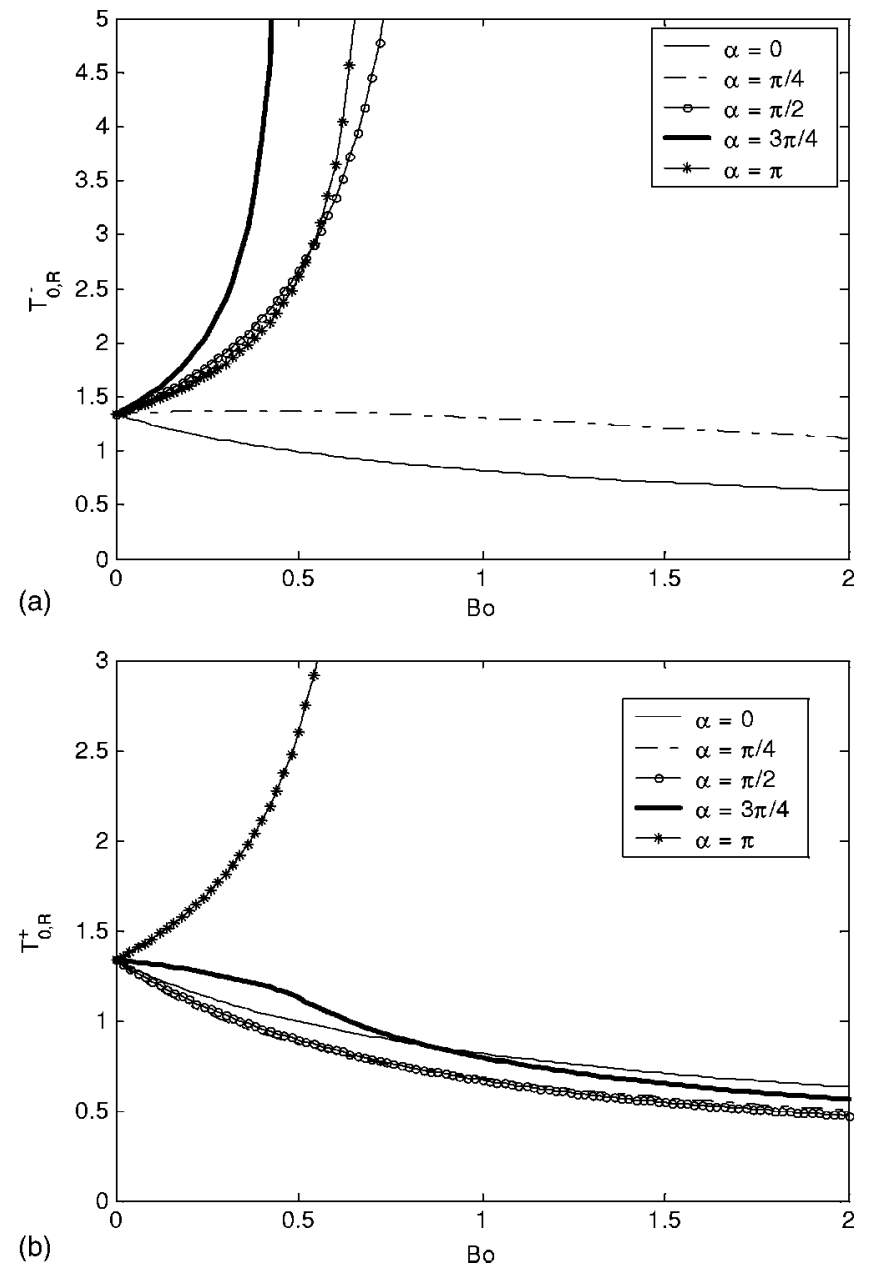

FIG. 4. Trailing film thickness in units of half-channel width vs $B o$ on the (a) lower and (b) upper wall at $O\left(\mathrm{Ca}^{0}\right)$.

In the front of the plug, the precursor film thickness $T_{0, F}^{ \pm}$ is a specified quantity and (27) serves as an equation for $A_{0}^{ \pm}$. Thus, to solve for the front transition regions, the constant $A$ in (25) is used as a shooting parameter that is iterated until (27) is satisfied for the particular choice of $T_{0, F}^{ \pm}$. If $h_{0, x x}^{ \pm}\left(x_{0, R}^{ \pm}\right)=0$ for the rear interface for some $\left(B o_{\mathrm{c}}, \alpha\right)$, then $h_{0, x x}^{ \pm}\left(x_{0, F}^{ \pm}\right)=0$ for the front interface for $\left(B o_{\mathrm{c}}, \pi-\alpha\right)$. Therefore when $\alpha \leqslant \pi / 2$ solutions for the front transition region only exist for $B o<B o_{\mathrm{c}}$.

\section{Higher-order corrections: Statics region}

It can be shown that the $O\left(\mathrm{Ca}^{1 / 3}\right)$ corrections to the interface shape and pressure, $h_{1}^{ \pm}$and $p_{1}$, are both equal to zero. As a result the coordinate translation in (24) is found from the matching conditions at $O\left(\mathrm{Ca}^{2 / 3}\right)$ to be

$$
X_{0}^{ \pm}=-\frac{A_{1}^{ \pm}}{A_{0}^{ \pm}} T_{0}^{ \pm}
$$

Since $h_{1}^{ \pm}=0$, the normal stress balance at $O\left(\mathrm{Ca}^{2 / 3}\right)$ in the statics region can be simplified to
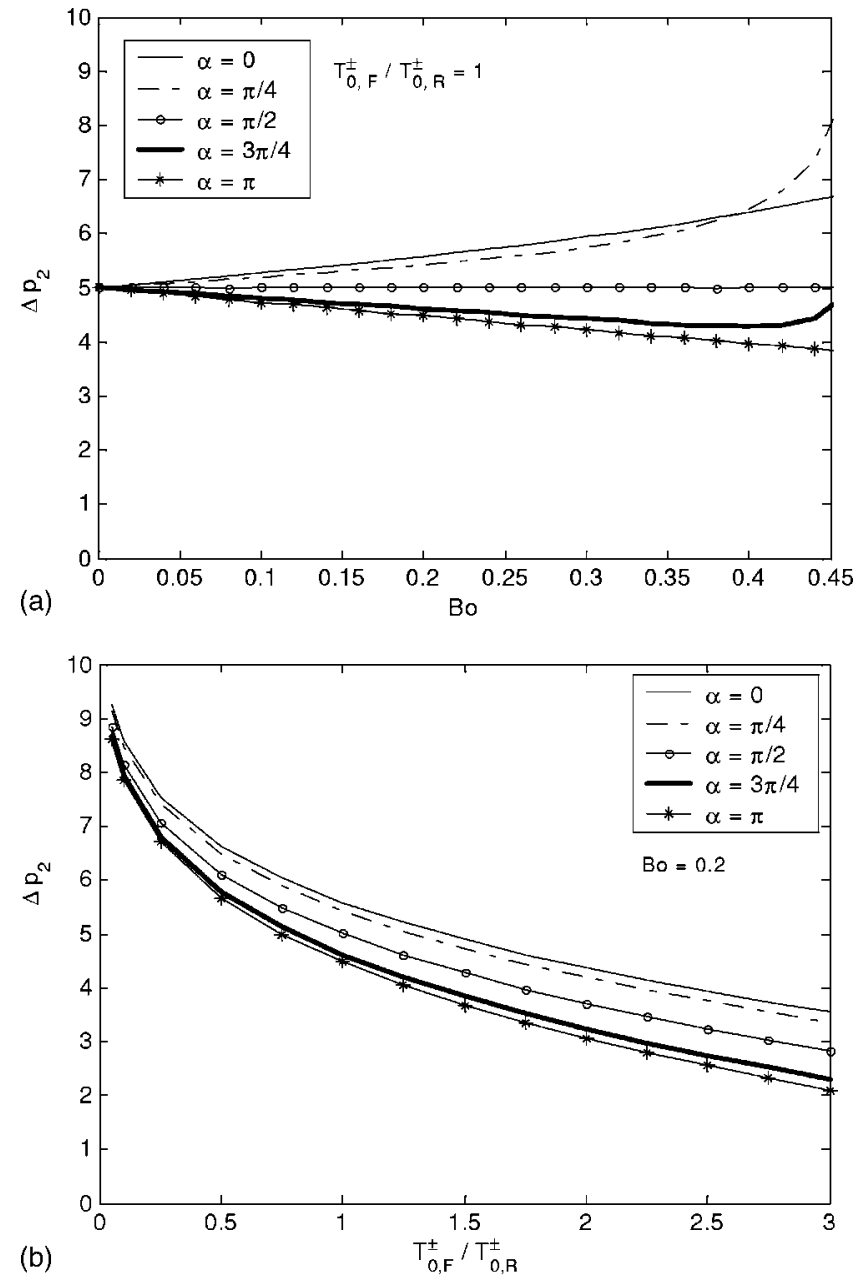

FIG. 5. Flow contribution to pressure drop across the plug as a function of (a) Bo and (b) precursor film thickness.

$$
\pm \frac{h_{2, x}^{ \pm}}{\left(1+h_{0, x}^{ \pm 2}\right)^{3 / 2}}=\delta p_{2}\left(x-x_{t}\right)
$$

with boundary conditions

$$
h_{2}^{ \pm}\left(x_{0}^{ \pm}\right)=-\frac{1}{h_{0, x x}^{ \pm}\left(x_{0}^{ \pm}\right)}\left(\frac{\left(A_{1}^{ \pm}\right)^{2}}{2}-A_{0}^{ \pm} A_{2}^{ \pm}\right),
$$

obtained by comparing terms independent of $X$ at $O\left(\mathrm{Ca}^{2 / 3}\right)$ in (17). As before, (29) and (30) are transformed to arc-length coordinates and integrated numerically to determine $\delta p_{2}$. Details can be found in the Appendix.

The pressure drop across the plug is

$$
\begin{aligned}
\Delta_{p} & =p_{\text {air }, R}-p_{\text {air }, F} \\
& =\left(\delta p_{0, R}+C a^{2 / 3} \delta p_{2, R}\right)-\left(\delta p_{0, F}+C a^{2 / 3} \delta p_{2, F}\right)+O(C a) \\
& =\Delta p_{0}+C a^{2 / 3} \Delta p_{2}+O(C a),
\end{aligned}
$$

where $\Delta p_{0}$ is the hydrostatic contribution and $\Delta p_{2}$ is the leading-order flow contribution. Since we are primarily interested in the effects of flow, we examine how $\Delta p_{2}$ depends on the system parameters. In Fig. 5(a) $\Delta p_{2}$ is plotted against $B o$ for different $\alpha$ with the precursor film thickness on the lower and upper walls equal to the corresponding trailing film thickness. This corresponds to steady plug propagation in 
which the plug volume does not change. For $\alpha<\pi / 2$ the pressure drop increases with $B o$. This is a result of greater viscous stresses associated with the decreasing film thickness at these orientations (see Fig. 3). When $\alpha=\pi / 2$ the thickening of the lower film exactly counteracts the thinning of the upper and the pressure drop remains constant. For $\alpha>\pi / 2$ the pressure drop initially decreases with $B o$, but eventually increases and diverges as the film thickness diverges. Figure 5(b) shows that at any orientation $\Delta p_{2}$ decreases as the pre- cursor film thickness increases. This is consistent with previous results ${ }^{32,34}$ that showed that it is easier to drive a plug through a tube with thicker precursor film.

\section{Higher-order corrections: Transition regions}

In the transition regions lubrication equations still apply at $O\left(\mathrm{Ca}^{1 / 3}\right)$ and the following third-order equation is found for the correction term $H_{1}^{ \pm}(X)$ :

$$
H_{1, X X X}^{ \pm}=-\frac{3\left(2 H_{0}^{ \pm}-3 T_{0}^{ \pm}\right) H_{1}^{ \pm}+3 T_{1}^{ \pm} H_{0}^{ \pm}+B o \cos (\alpha) H_{0}^{ \pm}\left(H_{0}^{ \pm^{3}}-T_{0}^{ \pm}{ }^{3}\right)}{H_{0}^{ \pm}} .
$$

Note that there is a contribution from the gravitational component of the pressure gradient in the $x$ direction, $B o \cos (\alpha)$. The expression found by Wilson ${ }^{41}$ for the dragout problem reduces to (32) after some simplification. The result of Park and Homsy ${ }^{21}$ is recovered when $B o=0$ or $\alpha=\pi / 2$. Equation (32) is rescaled as in (24) with $J_{1}^{ \pm}=H_{1}^{ \pm} / T_{0}^{ \pm}, \hat{T}_{1}^{ \pm}$ $=T_{1}^{ \pm} / T_{0}^{ \pm}, \xi=\left(X+X_{0}^{ \pm}\right) / T_{0}^{ \pm}$to get

$$
J_{1, \xi \xi \xi \xi}^{ \pm}=-\frac{3\left(2 J_{0}^{ \pm}-3\right) J_{1}^{ \pm}+3 \hat{T}_{1}^{ \pm} J_{1}^{ \pm}+B o \cos (\alpha) T_{0}^{ \pm} J_{0}^{ \pm}\left(J_{0}^{ \pm^{3}}-1\right)}{J_{0}^{ \pm}} .
$$

Initial conditions for integrating (33) are obtained from the asymptotic behavior in the vicinity of the thin films,

$$
\begin{aligned}
J_{1}^{ \pm} \sim \hat{T}_{1, R}^{ \pm}+A \exp (\lambda \xi), \quad \xi \rightarrow-\infty(\text { rear }), \\
J_{1}^{ \pm} \sim \hat{T}_{1, F}^{ \pm}+\exp (-\lambda \xi / 2)(A \cos (\sqrt{3} \lambda \xi / 2) \\
+B \sin (\sqrt{3} \lambda \xi / 2)), \quad \xi \rightarrow \infty \text { (front }),
\end{aligned}
$$

where $\lambda=3^{1 / 3}$ as before. Note that $\hat{T}_{1, R}^{ \pm}$is unknown in the rear, but $\hat{T}_{1, F}^{ \pm}$is specified in the front. Thus the initial conditions involve two unknown constants, $\left(\hat{T}_{1, R}^{ \pm}, A\right)$ in the rear and $(A, B)$ in the front, which are determined by matching the transition region and statics region solutions. In the vicinity of the statics region $J_{1}^{ \pm}$has the asymptotic form

$$
\begin{aligned}
& J_{1}^{ \pm} \sim-\left(B o \cos (\alpha)\left(T_{0}^{ \pm}\right)^{2} / 6\right) \xi^{3}+\left(B_{0}^{ \pm} / 2\right) \xi^{2}+B_{1}^{ \pm} \xi+B_{2}^{ \pm}, \\
& \xi \rightarrow \pm \infty .
\end{aligned}
$$

Comparing terms involving $X^{3}, X^{2}$, and $X$ in the $O(C a)$ matching condition we find

$$
\begin{aligned}
& h_{0, x x x}^{ \pm}\left(x_{0}^{ \pm}\right)= \pm B o \cos (\alpha), \\
& h_{1, x x}^{ \pm}\left(x_{0}^{ \pm}\right)=\left(\frac{B_{0}^{ \pm}}{2 T_{0}^{ \pm}}-\frac{B o \cos (\alpha) X_{0}^{ \pm}}{2}\right),
\end{aligned}
$$

$$
h_{2, x}^{ \pm}\left(x_{0}^{ \pm}\right)=\mp\left(B_{1}^{ \pm}+\frac{B_{0}^{ \pm} X_{0}^{ \pm}}{T_{0}^{ \pm}}-\frac{B o \cos (\alpha) X_{0}^{ \pm^{2}}}{2}\right) .
$$

Equation (36) can be obtained from (19) and (21) and provides no new information. Since $h_{1}^{ \pm}, h_{2}^{ \pm}, T_{0}^{ \pm}$, and $X_{0}^{ \pm}$are known, (37) and (38) and provide boundary conditions for (33), which is solved by numerical integration using (34) as the initial condition and choosing $\left(\hat{T}_{1, R}^{ \pm}, A\right)$ or $(A, B)$ to satisfy (37) and (38). Figures 6(a) and 6(b) show the $O(\mathrm{Ca})$ correction to the trailing film thickness at the lower and upper walls, $T_{1, R}^{-}$and $T_{1, R}^{+}$, as a function of $B o$ for different $\alpha$. When $\alpha<\pi / 2$ both $T_{1, R}^{-}$and $T_{1 R}^{+}$are negative, indicating that this correction makes the film thinner. The opposite is true when $\alpha>\pi / 2$. In the former case, $T_{1, R}$ and $T_{1, R}^{+}$have slight minima for some orientations, while in the latter $T_{1, R}^{+}$passes through a maximum for $\alpha=3 \pi / 4$. Since (33) involves $T_{0}^{ \pm}$ which diverges at $B o=B o_{\mathrm{c}}, T_{1, R}^{ \pm}$also diverges at $B o_{\mathrm{c}}$. When $B o=0$ or $\alpha=\pi / 2$ the gravitational term involving $B o \cos (\alpha)$ disappears from (33) and the boundary conditions, (37) and (38). As a result $T_{1, R_{21}^{ \pm}}^{ \pm}=0$, which is consistent with the results of Park and Homsy.

\section{THE GRAVITATIONAL REGIME}

The analysis in Sec. III breaks down when the curvature of the static solution at the lower wall becomes zero and the film thickness diverges. This occurs when $\alpha \geqslant \pi / 2$ for the rear meniscus and $\alpha \leqslant \pi / 2$ for the front meniscus. In this section we determine the critical Bond number $B o_{\mathrm{c}}$ for breakdown and examine the structure of the solution in the lower transition region where this occurs. This analysis applies to either the rear or front interface for appropriate values of $\alpha$, while the viscocapillary analysis applies to the other interface.

\section{A. Critical Bond number}

Since the curvature in the lower transition region becomes small at $B o=B o_{c}$, the gravitational scaling (14) applies in this region while the viscocapillary scaling (11) applies in the upper transition region. The perturbation 


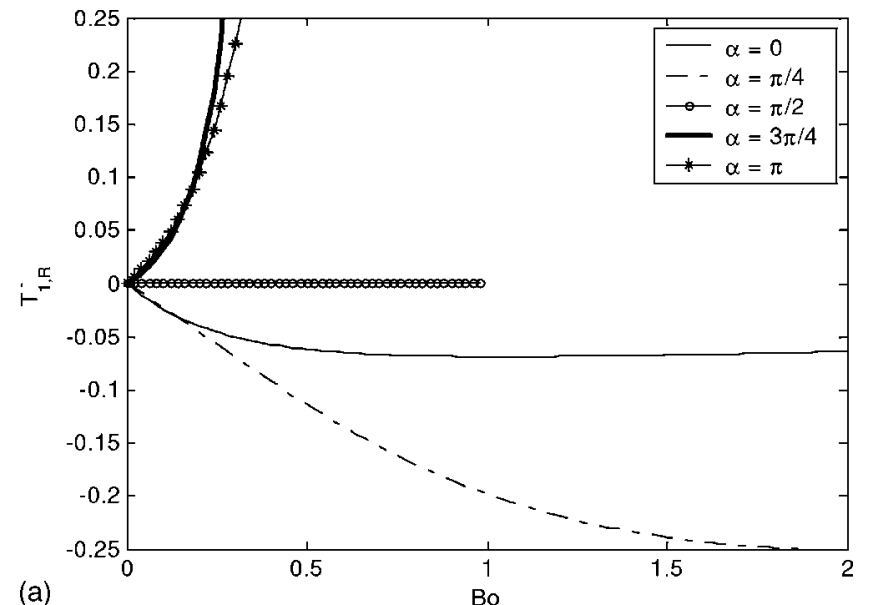

(a)

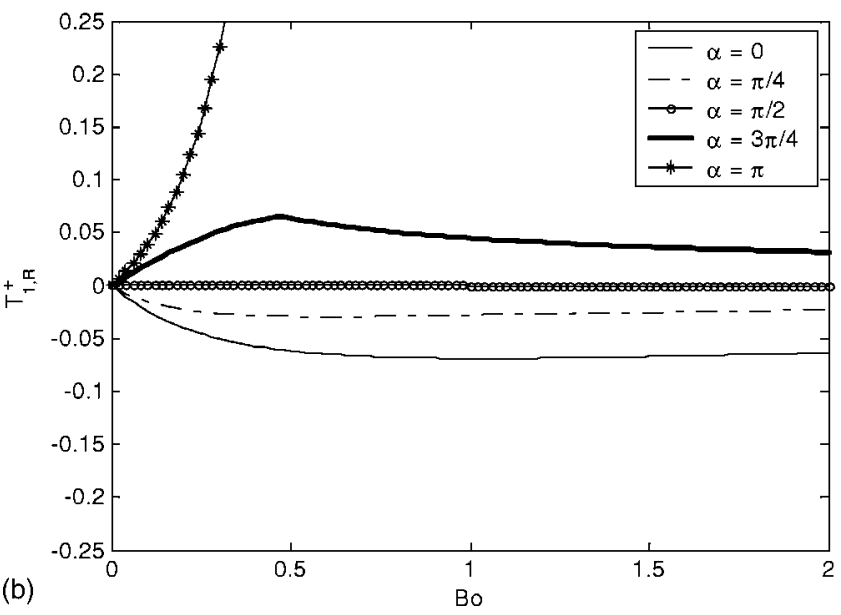

(b)

FIG. 6. Trailing film thickness in units of half-channel width vs $B o$ on the (a) lower and (b) upper wall at $O\left(\mathrm{Ca}^{1 / 3}\right)$.

expansion (15) for both regions proceeds in powers of $\mathrm{Ca}^{1 / 6}$. In the statics region, the normal stress condition is identical to (19) at leading order and is reproduced below:

$$
B o_{\mathrm{c}} x \cos (\alpha)-B o_{\mathrm{c}} h_{0}^{ \pm}(x) \sin (\alpha)-\delta p_{0}= \pm \frac{h_{0, x x}^{ \pm}}{\left(1+{h_{0, x}^{ \pm}}^{2}\right)^{3 / 2}}
$$

Boundary conditions are obtained by considering terms involving $h_{0}^{ \pm}(x)$ up to $O\left(\mathrm{Ca}^{1 / 3}\right)$ in the matching condition (17),

$$
\begin{array}{r}
O\left(\mathrm{Ca}^{0}\right): h_{0}^{ \pm}\left(x_{0}^{ \pm}\right)= \pm 1, \\
O\left(\mathrm{Ca}^{1 / 6}\right): h_{0, x}^{-}\left(x_{0}^{-}\right)=0, \\
O\left(\mathrm{Ca}^{1 / 3}\right): h_{0, x}^{+}\left(x_{0}^{+}\right)=0 \\
h_{0, x x}^{-}\left(x_{0}^{-}\right)=0 .
\end{array}
$$

Equations (40), (41), and (42a) are identical to (20) and (21), but $(42 b)$ is an additional condition that requires the curvature of the statics solution to vanish at the lower wall and provides a constraint to determine $B o_{\mathrm{c}}$. The system is transformed to arc-length coordinates as explained in the Appendix and $B o_{\mathrm{c}}$ is determined as a function of $\alpha$. Results are

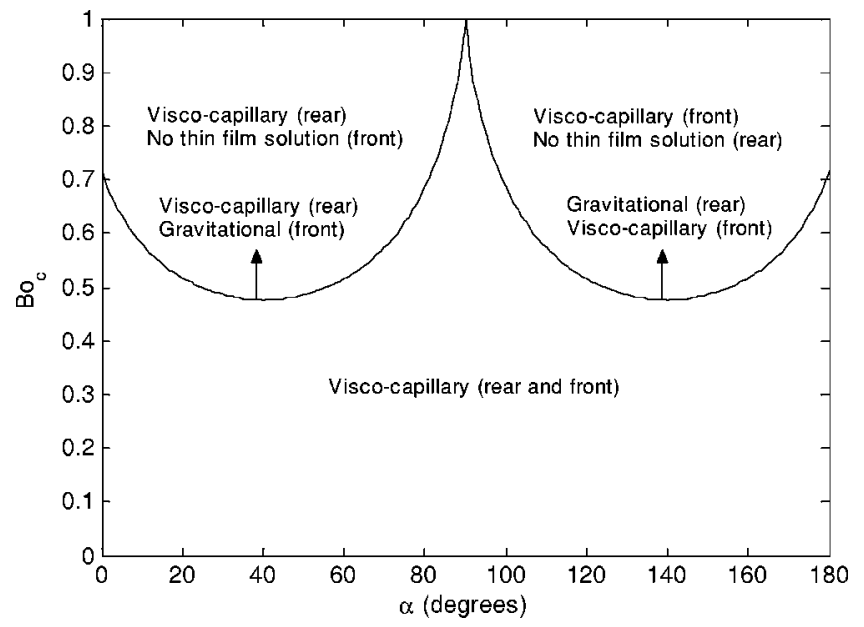

FIG. 7. Critical Bond number $B o_{\mathrm{c}}$ for different orientations. For $B o<B o_{\mathrm{c}}$ the viscocapillary scaling (11) is valid in all transition regions. In the lower transition regions, for $B o=B o_{\mathrm{c}}+B C a^{1 / 6}$ and $\alpha>\pi / 2$, the gravitational scaling (14) holds behind the plug and the viscocapillary scaling holds in front of the plug-these scalings are reversed when $\alpha<\pi / 2$. The upper transition regions follow the viscocapillary scaling for all $B o$ (except for $\alpha=0, \pi$ when $B o \geqslant B o_{\mathrm{c}}$ ), however corresponding to $\alpha<\pi / 2$ or $\alpha>\pi / 2$, this scaling applies either to the rear or the front meniscus, and the opposite meniscus has no thin film solution.

plotted in Fig. 7. It is seen that $B o_{\mathrm{c}}$ has two minima at $\alpha$ $\sim \pi / 4,3 \pi / 4$, and a maximum value of 1 at $\alpha=\pi / 2$.

\section{B. $O\left(\mathrm{Ca}^{0}\right)$ equations in the transition region}

The upper transition region is described by (23). Rescaling variables in the lower transition region leads to lubrication equations, which are solved to obtain the following equation for the interface location:

$$
J_{0, \xi \xi \xi}=\frac{3\left(J_{0}^{-}-1\right)}{\left(J_{0}^{-}\right)^{3}}-\frac{B o_{\mathrm{c}} \cos (\alpha)\left(T_{0}\right)^{2}\left(\left(J_{0}^{-}\right)^{3}-1\right)}{\left(J_{0}^{-}\right)^{3}},
$$

where $J_{0}^{-}=H_{0}^{-} / T_{0}^{-}, \xi=\left(X+X_{0}^{-}\right) / T_{0}^{-}$as in (24). This equation with $\alpha=0$ was obtained by Derjaguin ${ }^{40}$ to describe the thickness of the liquid film on a plate withdrawn from a fluid reservoir. In the context of that problem, different approximations have been used to find solutions of (43) that match to the flat fluid interface in the reservoir. ${ }^{43,44}$ Here we describe a solution that uses exact matching conditions to connect the film to the plug in the statics region.

In the vicinity of the thin films $J_{0}^{-}$has the asymptotic form

$$
J_{0}^{-} \sim 1+A \exp (\lambda \xi), \quad \xi \rightarrow \infty(\text { rear/front }),
$$

and $\lambda$ satisfies

$$
\lambda^{3}-3\left(1-\left(T_{0}\right)^{2} B o_{\mathrm{c}} \cos (\alpha)\right)=0 .
$$

For the rear transition region, (43) is valid when $\alpha>\pi / 2$. In this case the single real root of (45), $\lambda=\lambda_{R}=[3(1$ $\left.\left.-\left(T_{0}^{-}\right)^{2} B o_{\mathrm{c}} \cos (\alpha)\right)\right]^{1 / 3}$, is positive and initial conditions that decay as $\xi \rightarrow-\infty$ can be found. The constant $A$ is arbitrarily set equal to 1 because of the translation $X_{0}^{-}$. In contrast to the viscocapillary regime, the unknown film thickness $T_{0}^{-}$explicitly appears in the equation and initial conditions. It is there- 
fore treated as a shooting parameter and determined by matching $J_{0}^{-}$to the statics region, where it has the asymptotic form

$$
\begin{aligned}
& J_{0}^{-} \sim-\frac{B o_{\mathrm{c}} \cos (\alpha)\left(T_{0}^{-}\right)^{2} \xi^{3}}{6}+\frac{A_{0}^{-} \xi^{2}}{2}+A_{1}^{-} \xi+A_{2}^{-}, \\
& \xi \rightarrow \pm \infty \text { (rear/front) } .
\end{aligned}
$$

Rewriting (46) in terms of $X$ and matching to appropriate terms at $O\left(\mathrm{Ca}^{1 / 2}\right)$ in the Taylor series expansion of $h^{-}(x)$ we find

$$
\begin{aligned}
& h_{0, x x x}^{-}\left(x_{0}^{-}\right)= \pm B o_{\mathrm{c}} \cos (\alpha), \\
& h_{1, x x}^{-}\left(x_{0}^{-}\right)=\left(\frac{A_{0}^{-}}{T_{0}^{-}}-B o_{\mathrm{c}} \cos (\alpha) X_{0}^{-}\right), \\
& h_{2, x}^{-}\left(x_{0}^{-}\right)=\left(A_{1}+\frac{A_{0} X_{0}^{-}}{T_{0}^{-}}-\frac{B o_{\mathrm{c}} \cos (\alpha) X_{0}^{-2}}{2}\right) .
\end{aligned}
$$

Equation (47) follows from (39) and (41) and does not provide new information. Thus higher-order corrections in the statics region, $h_{1}^{-}(x)$ and $h_{2}^{-}(x)$, are required to complete the transition region solution. It can be shown that $h_{1}^{ \pm}(x)$ and $h_{2}^{ \pm}(x)$ satisfy homogeneous equations and boundary conditions and are equal to zero. Then (48) and (49) provide conditions to determine $T_{0}^{-}$and $X_{0}^{-}$and complete the solution for $J_{0}^{-}$. Figure 8(a) shows $T_{0}^{-}$as a function of $\alpha$ when $B o=B o_{\mathrm{c}}$. Note that (43) reduces to the Landau-Levich equation, (23), when $\alpha=\pi / 2$ and (48) indicates that $T_{0}^{-}$diverges unless $A_{0}^{-}$ $=0$. It was not possible to find a solution with $A_{0}^{-}=0$ for $\alpha$ $=\pi / 2$. This case has been treated by Jensen et al. ${ }^{35}$ and we will discuss their results later in this section.

In the front transition region, (43) applies for $\alpha<\pi / 2$. If $\left(T_{0, F}\right)^{2} B o_{\mathrm{c}} \cos (\alpha)<1$, (45) has two roots with negative real part, $\lambda=\lambda_{R}(-1 \pm i \sqrt{3}) / 2$, which provide decaying solutions as $\xi \rightarrow+\infty$ and a solution for $J_{0}^{-}$can be found for each choice of $T_{0, F}$. On the other hand, if $\left(T_{0, F}^{-}\right)^{2} B o_{\mathrm{c}} \cos (\alpha)>1$ there is a single root with negative real part, $\lambda=\lambda_{R}$. Treating $T_{0, F}$ as an unknown quantity the procedure used for the rear transition region was followed; however no solutions satisfying (48) and (49) could be found. Thus there is an upper limit to the precursor film thickness for which solutions of the front transition region exist, and within this limit $T_{0, F}^{-}$can be chosen arbitrarily.

\section{Higher-order corrections: Statics region}

Since $h_{1}^{ \pm}(x)=h_{2}^{ \pm}(x)=0$, the normal stress balance in the statics region at $O\left(\mathrm{Ca}^{1 / 2}\right)$ can be simplified to

$$
\pm \frac{h_{3, x}^{ \pm}}{\left(1+h_{0, x}^{-2}\right)^{3 / 2}}=\delta p_{3}\left(x-x_{t}\right) \text {. }
$$

Matching terms independent of $X$ at $O\left(\mathrm{Ca}^{1 / 2}\right)$ in (17) yields
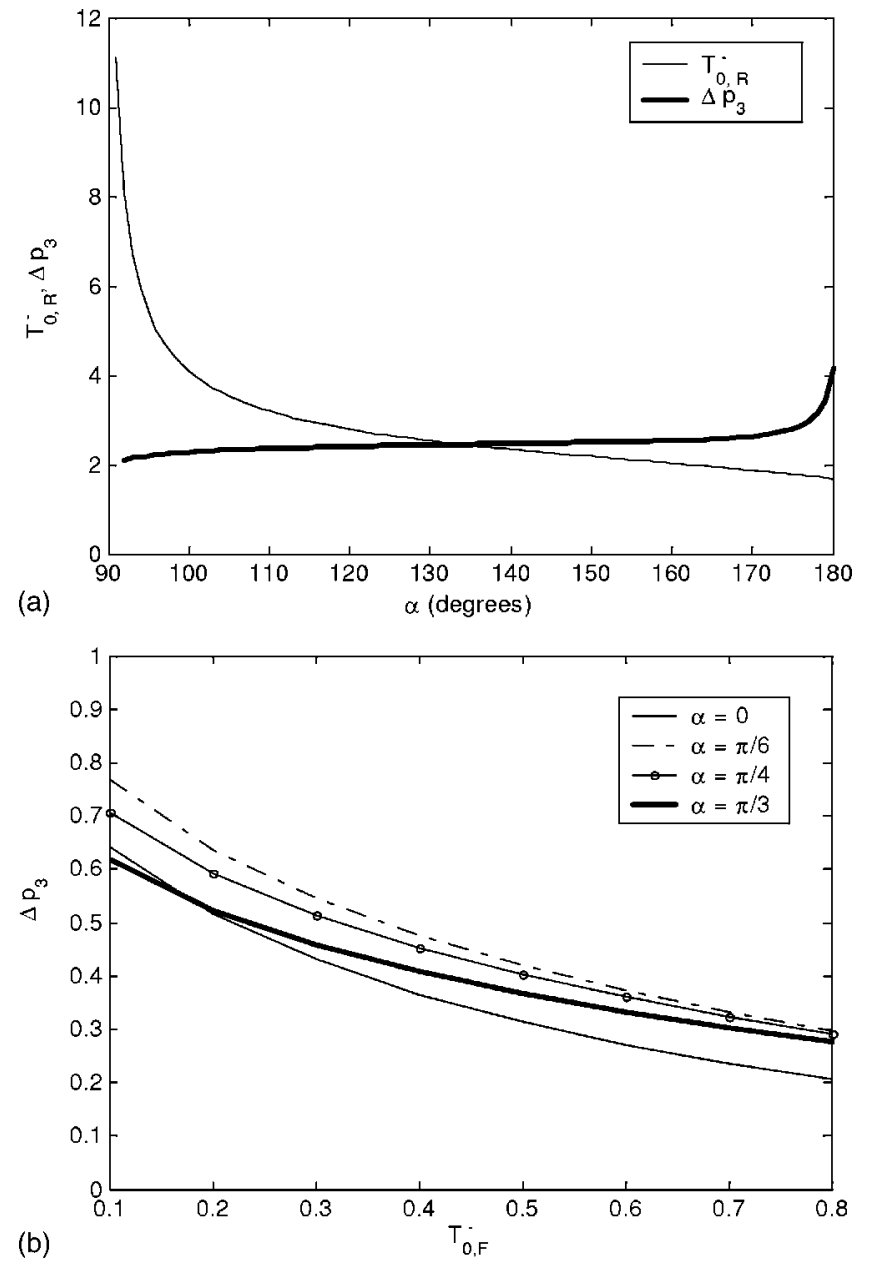

FIG. 8. (a) Trailing film thickness and flow contribution to pressure drop across rear meniscus at $B o=B o_{\mathrm{c}}$ and (b) flow contribution to pressure drop across front meniscus as a function of precursor film thickness at $B o=B o_{\mathrm{c}}$.

$$
\begin{aligned}
h_{3}^{-}\left(x_{0}^{-}\right)= & \mp\left(A_{2} T_{0}^{-}+A_{1} X_{0}^{-}+\frac{A_{0}\left(X_{0}^{-}\right)^{2}}{2 T_{0}^{-}}\right. \\
& \left.-\frac{B o_{\mathrm{c}} \cos (\alpha)\left(X_{0}^{-}\right)^{3}}{6}\right)
\end{aligned}
$$

where all the quantities on the right side are known. At the upper wall matching conditions of the viscocapillary regime apply and lead to the homogeneous condition

$$
h_{3}^{+}\left(x_{0}^{+}\right)=0 \text {. }
$$

Equations (51) and (52) specify boundary conditions for $h_{3}^{+}(x)$ and $h_{3}^{-}(x)$ and the requirement that they join smoothly at $x=x_{t}$ determines $\delta p_{3}$. When $\alpha=0$ or $\pi$, the symmetry condition $h_{3}^{-}\left(x_{t}\right)=0$ is used along with either (51) (for the rear interface when $\alpha=\pi$; front interface when $\alpha=0$ ) or (52) (for the rear interface when $\alpha=0$; front interface when $\alpha=\pi$ ). The flow contribution to the pressure drop across the plug is dominated by the $O\left(\mathrm{Ca}^{1 / 2}\right)$ contribution from either the rear or front meniscus, since the drop across the other meniscus is $O\left(\mathrm{Ca}^{2 / 3}\right) . \delta p_{3}$ for the rear meniscus is plotted in Fig. 8(a) versus $\alpha$ and increases with $\alpha$ as the film becomes thinner. 
For $\alpha<\pi / 2$, Fig. 8(b) shows $\delta p_{3}$ across the front meniscus as a function of $T_{0, F}^{-}$. As before $\delta p_{3}$ decreases with increasing $T_{0, F}$.

It remains to discuss the structure of the solution for $B o>B o_{c}$. For small increments over $B o_{c}$, the Bond number can be expanded in powers of $C a^{1 / 6}$, i.e., $B o=B o_{c}+B C a^{1 / 6}$, $B=O(1)>0$; and the preceeding approach applies. In this case, the $O\left(\mathrm{Ca}^{1 / 6}\right)$ and $O\left(\mathrm{Ca}^{1 / 3}\right)$ statics solutions, $h_{1}^{ \pm}(x)$ and $h_{2}^{ \pm}(x)$, will be nonzero and modify the matching conditions that determine the film thickness. The flow contribution to the pressure drop will be $O\left(\mathrm{Ca}^{1 / 6}\right)$ while the film thickness remains $O\left(\mathrm{Ca}^{1 / 2}\right)$. We do not present such an analysis here, but only note that a consistent asymptotic solution can be found.

For higher $B o$, when the system (39)-(42) was solved by relaxing (40), the interface did not contact the lower wall, but passed through an inflection point at $h_{0}^{-}\left(x_{0}^{-}\right)=y_{1},-1$ $<y_{1}<1$. The origin of the transition region is thus located at a distance $\left(1+y_{1}\right)$ above the lower wall and the film thickness is $O(1)$. However, a consistent solution cannot be found because the viscous and body force terms do not balance each other in the $x$ momentum equation in the lower film,

$$
C a \frac{\partial^{2} u}{\partial y^{2}}+B o \cos (\alpha)=0,
$$

if the film thickness is $O(1)$ and $C a \ll 1$. This inconsistency is the result of neglecting the viscous terms in the normal stress condition that are comparable in size to the other terms in the vicinity of the inflection point. Thus it is necessary to solve for the velocity field and the asymptotic approach breaks down. An exception is the special case $\alpha=\pi / 2$ for which the gravitational term vanishes in (53). For $B o \geqslant B o_{c}$, it can be shown that all derivatives of $h_{0}^{-}(x)$ vanish at $y=y_{1}$, the transition region vanishes and the static interface joins smoothly to a film of thickness $\left(1+y_{1}\right)$ that is independent of the capillary number. ${ }^{35}$

\section{DISCUSSION}

To discuss the application of these results to human airways, we examine the effect of gravity and propagation speed on two features of physiological significance, namely, prebifurcation asymmetry in the liquid distribution and plug rupture. We estimate values for the dimensionless parameters for two cases based on physical properties and airway dimensions and speeds from Cassidy et al. ${ }^{18}$ and King et al..$^{47}$ (1) SRT using the surfactant Survanta $\left[\rho=1.16 \mathrm{~g} / \mathrm{cm}^{3}, \mu\right.$ $=0.52 \mathrm{~g} /(\mathrm{cm} \mathrm{s})$, and $\sigma=48 \mathrm{dyn} / \mathrm{cm}]$ and (2) PLV using perflurorcarbon liquids $\left[\rho=1.93 \mathrm{~g} / \mathrm{cm}^{3}, \mu=0.021 \mathrm{~g} /(\mathrm{cm} \mathrm{s})\right.$, and $\sigma=18 \mathrm{dyn} / \mathrm{cm}]$. Under these conditions Bond and capillary numbers vary in the range $0.01<B o<0.2,0.01<C a<0.1$ (SRT); $0.04<B o<0.6,0.001<C a<0.01$ (PLV) between generations 11 and 17 of the adult human lung. In the discussion to follow we use the results of the viscocapillary regime over the parameter range $0.0<B o<0.4,0.0<C a$ $<0.05$ which is relevant to SRT and PLV in small airways.

\section{A. Prebifurcation asymmetry}

A composite solution for the shape of the interface accurate to $O\left(\mathrm{Ca}^{2 / 3}\right)$ was obtained by using the method of additive composition, ${ }^{48}$

$$
\begin{aligned}
h_{c}= & h_{0}^{ \pm}(x)+C a^{2 / 3} h_{2}^{ \pm}(x) \pm\left[1-C a^{2 / 3}\left(H_{0}^{ \pm}(X)\right.\right. \\
& \left.\left.+C a^{1 / 3} H_{1}^{ \pm}(X)\right)\right]- \text { c.p. },
\end{aligned}
$$

where the common part c.p. was determined from the matching conditions to be

$$
\begin{aligned}
c . p .= & h_{0}^{ \pm}\left(x_{0}^{ \pm}\right)+h_{0, x x}^{ \pm}\left(x_{0}^{ \pm}\right) \frac{\left(x-x_{0}^{ \pm}\right)^{2}}{2}+h_{0, x x x}^{ \pm}\left(x_{0}^{ \pm}\right) \frac{\left(x-x_{0}^{ \pm}\right)^{3}}{6} \\
& +C a^{2 / 3}\left[h_{2}^{ \pm}\left(x_{0}^{ \pm}\right)+h_{2, x}^{ \pm}\left(x_{0}^{ \pm}\right)\left(x-x_{0}^{ \pm}\right)\right] .
\end{aligned}
$$

Sample interface shapes for different parameter values are graphed in Fig. 9. In this figure and the results to follow, the precursor film thickness on either wall, $h_{\infty, F}^{ \pm}$, is fixed at 0.1 . In each panel the interface for $B o=0$, which is symmetric about the centerline of the channel $(y=0)$, is shown for comparison. When $B o>0$, the interface is distorted as more liquid pools below the centerline $(y<0)$. The asymmetry increases with increasing $C a$ and depends on $\alpha$ : The shape of the rear interface is most sensitive to changes in $\mathrm{Bo}$ and $\mathrm{Ca}$ for $\alpha>\pi / 2$, while the front interface is most sensitive for $\alpha<\pi / 2$. Since the precursor film thickness is independent of $\mathrm{Bo}$ and $\mathrm{Ca}$, the shape of the front interface does not vary as much as that of the rear.

An interesting feature of the interface shape is the "necking in" seen at the front meniscus. The nonmonotonic shape represents a capillary wave at the interface and is a result of the oscillatory initial conditions (25) and (34) required for the solution of (23) and (33), respectively. Such profiles have been described by other authors for the motion of long bubbles $^{20}$ and plugs in tubes. ${ }^{32,34}$ The capillary wave causes the minimum distance between the interface and the channel wall, $h_{\min }$, to be less than the constant film thickness far from the plug. The dependence of $h_{\min }$ on $B o$ and $\alpha$ mirrors the variation of the trailing film thickness with these parameters: on the lower wall, $h_{\min }$ passes through a slight maximum as $B o$ is increased for $\alpha=\pi / 4$, whereas it decreases monotonically with $B o$ for $\alpha=\pi / 2,3 \pi / 4$. On the upper wall, $h_{\min }$ decreases monotonically with $B o$ for $\alpha=\pi / 4, \pi / 2,3 \pi / 4$.

When the plug splits at an airway bifurcation, the asymmetric liquid distribution can play a role in determining the volume of liquid delivered to each downstream branch (see Fig. 1). In order to quantify the prebifurcation asymmetry, a volume ratio $V_{R}$ is defined as the ratio of the liquid volume above the centerline of the channel to that below per unit width of the channel and is equal to

$$
V_{R}=\frac{V^{+}+L_{P}}{V^{-}+L_{P}}
$$

where $L_{P}$ is the plug length and

$$
\begin{aligned}
V^{+}= & \int_{x_{1, R}^{+}}^{x_{2, R}} h_{c, R}(x) d x+\int_{x_{2, F}}^{x_{1, F}^{+}} h_{c, F}(x) d x+\left(x_{t}-x_{2, R}\right) \\
& +\left(x_{2, F}-x_{t}\right),
\end{aligned}
$$



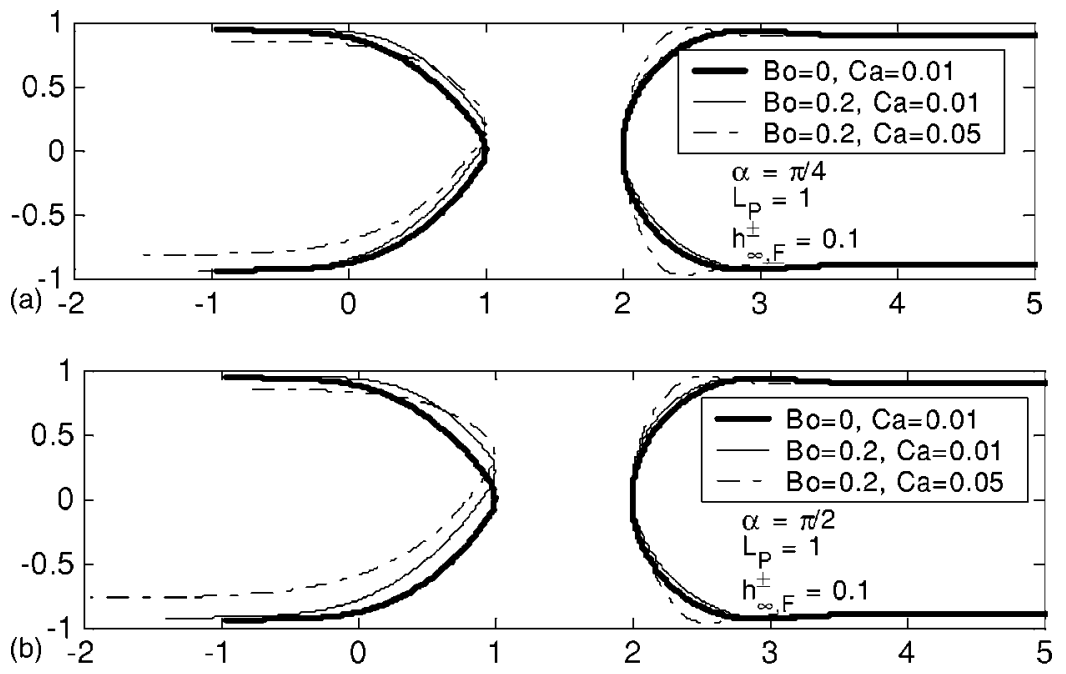

FIG. 9. Composite solution showing the shape of the plug for three combinations of $\mathrm{Bo}$ and $\mathrm{Ca}$ at (a) $\alpha$ $=\pi / 4$, (b) $\alpha=\pi / 2$, and (c) $\alpha=3 \pi / 4$. Other parameter values are fixed at $h_{\infty, \mathrm{F}}^{ \pm}=0.1, L_{p}=1$. The interface for $B o=0$ (dotted line) in each panel is symmetric about $y=0$, but becomes distorted as $\mathrm{Bo}$ and $\mathrm{Ca}$ increase.

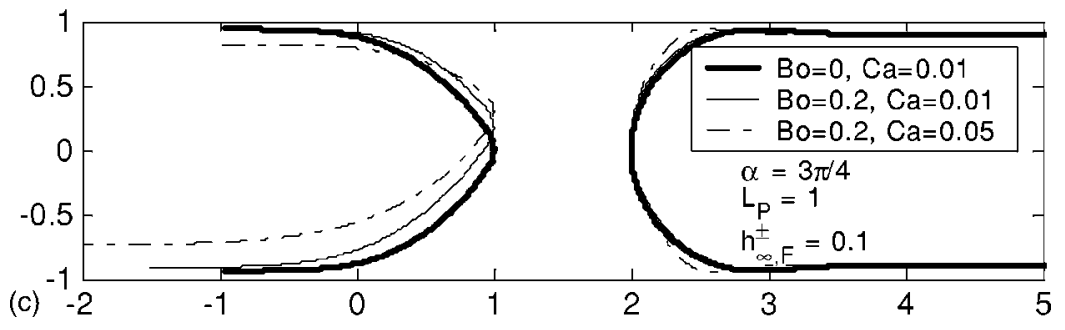

$$
\begin{aligned}
V^{-}= & -\int_{x_{1, R}^{-}}^{x_{2, R}} h_{c, R}(x) d x-\int_{x_{2, F}}^{x_{1, F}^{-}} h_{c, F}(x) d x+\left(x_{t}-x_{2, R}\right) \\
& +\left(x_{2, F}-x_{t}\right) .
\end{aligned}
$$

In (57), $x_{2, R / F}$ and $x_{t}$ are the locations corresponding to the centerline $\left(y=h_{c}=0\right)$ and "tip" $\left(d h_{c} / d x \rightarrow \infty\right)$, respectively. The other limits of integration, $x_{1, R / F}^{ \pm}$, are chosen to be the locations at which the distance between the wall and the interface reaches within $1 \%$ of the constant film thickness.

Figures 10 and 11 show the dependence of $V_{R}$ on different parameters. In Fig. 10(a), $V_{R}$ is plotted as a function of $B o$ for different orientations with $C a=0.01, L_{p}=1$. When $B o=0$, the plug is symmetric about $y=0$, and $V_{R}=1$, indicating symmetric liquid distribution on either side of the centerline. As $B o$ increases, a progressively larger fraction of the plug volume resides in the lower half of the channel $(y$ $<0)$ and $V_{R}$ decreases. The $B o$ dependence strongly depends on the orientation $\alpha$, and for $\alpha \geqslant \pi / 2, V_{R}$ decreases rapidly, leading to crossovers between the different curves.

Figure 10(b) shows that $V_{R}$ depends strongly on $C a$ and decreases as $\mathrm{Ca}$ is increased. The decrease is more pronounced at higher $B o$. When $C a=0$, the channel walls are dry and $V_{R}$ is calculated from the static solution. $V_{R}$ is less than 1 due to gravitational distortion of the static interface, and this effect increases with $B o$ When $C a>0$, dynamic effects enhance the gravitational distortion of the interface as seen in Fig. 9 and lead to a decrease in $V_{R}$. These results indicate that at low $\mathrm{Ca}$ in small airways higher propagation speeds lead to more asymmetric plug shapes. When such plugs split at airway bifurcations, the greater liquid volume below the centerline may enhance the amount of liquid delivered to the gravity-preferred branch and thus lead to more nonhomoge-
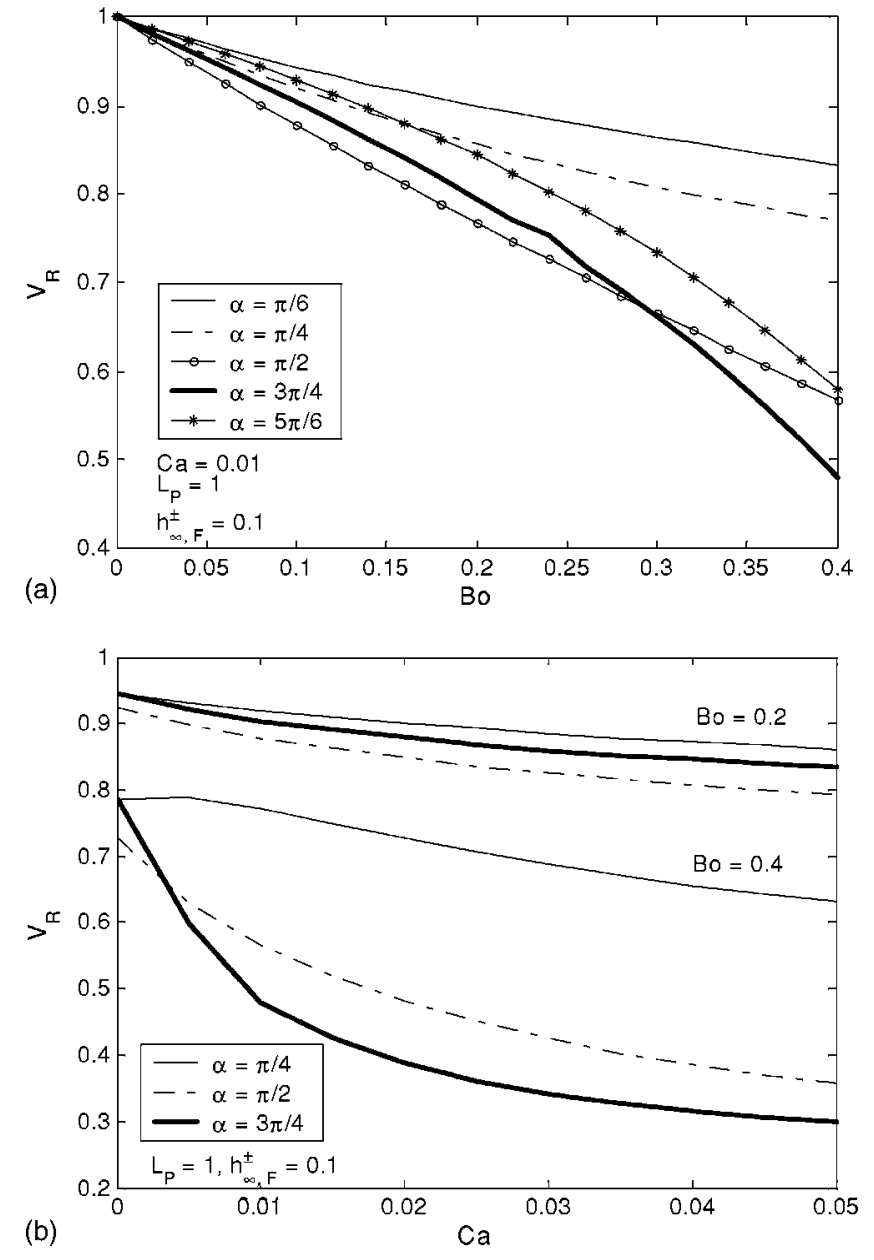

FIG. 10. Ratio, $V_{R}$, of liquid volumes per unit channel width above and below centerline, as a function of (a) $\mathrm{Bo}$ and (b) $\mathrm{Ca}$ for $L_{p}=1$ and different values of $\alpha$. 

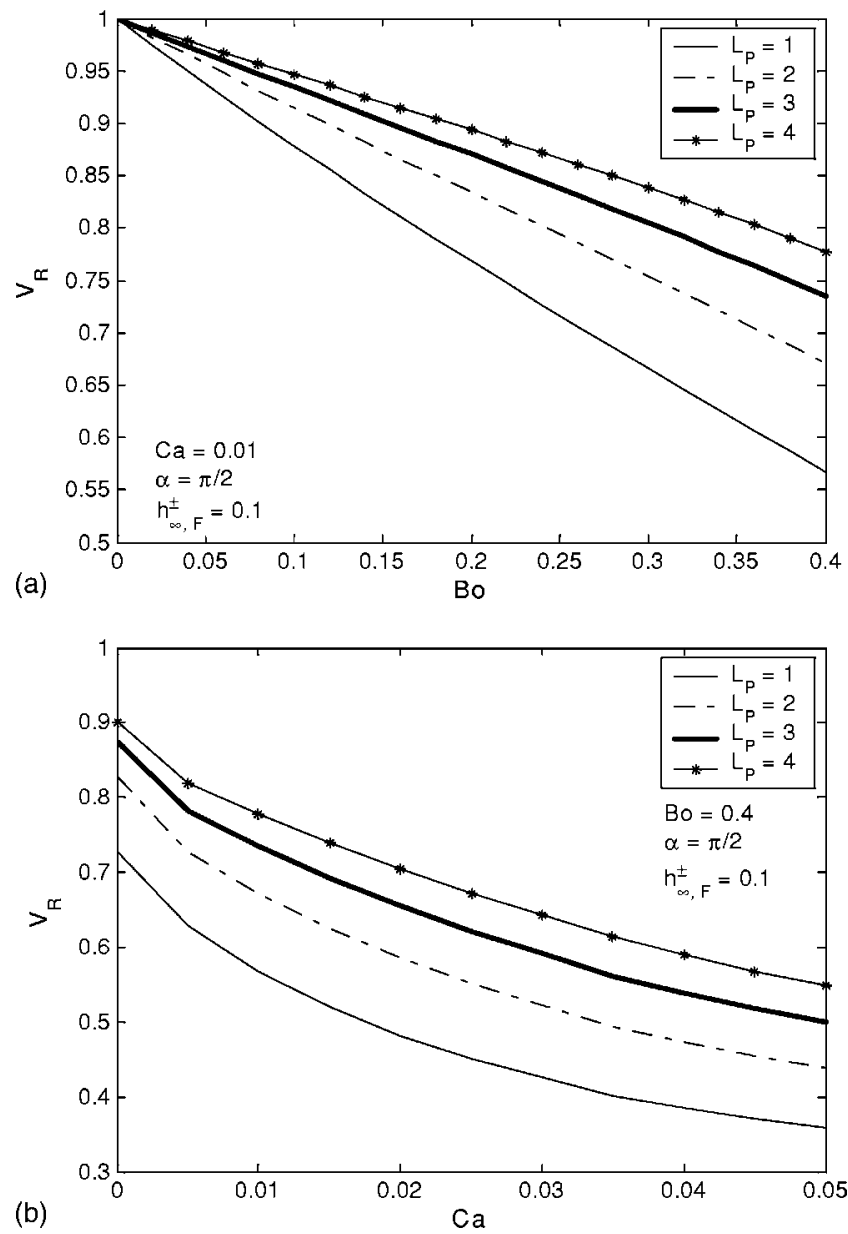

FIG. 11. Ratio, $V_{R}$, of liquid volumes per unit channel width above and below centerline, as a function of (a) $\mathrm{Bo}$ and (b) $\mathrm{Ca}$ for $\alpha=\pi / 2$ and different values of $L_{p}$.

neous liquid distribution. This is in contrast to the expected effect of flow in large airways, in which inertial effects could be important due to higher speeds and larger dimensions. Increasing the flow rate under such conditions would counter gravitational effects, leading to more homogeneous plug splitting if the airway bifurcation is geometrically symmetric.

Finally, we consider the effect of plug length $L_{p}$. Equation (56) indicates, and Fig. 11 confirms, that $V_{R}$ increases with $L_{p}$ as the liquid volume between the tips of the two interfaces increases. Thus the liquid distribution, and hence plug splitting, is likely to be more uniform for longer plugs. However, it must be noted that the asymptotic analysis is only valid for $L_{p}=O(1)$ since it neglects the fluid mechanical coupling and viscous pressure drop between the front and rear meniscus. These effects become important for very short and very long plugs, respectively.

\section{B. Plug rupture}

As the plug moves through the channel its volume decreases if the trailing film is thicker than the precursor film and it eventually ruptures. The instantaneous rate of volume change of the plug is determined by computing the volume flux in the thin films. The velocity profile in the thin films is parabolic since the $x$ momentum equation and boundary conditions reduce to

$$
\begin{aligned}
& C a \frac{\partial^{2} u}{\partial y^{2}}+B o \cos (\alpha)=0, \\
& u(y= \pm 1)=-1, \quad \frac{\partial u}{\partial y}\left(y= \pm\left(1-h_{\infty}^{ \pm}\right)\right)=0 .
\end{aligned}
$$

Then the flow rate per unit width of the channel in the precursor films is computed to be

$$
Q_{F}^{ \pm}=\frac{Q_{F}^{*^{ \pm}}}{(\mu / \sigma a)}=-C a h_{\infty, F}^{ \pm}+\frac{B o \cos (\alpha) h_{\infty, F}^{ \pm^{3}}}{3},
$$

where the first term represents the constant speed plug motion and the second gravitational drainage. Using the perturbation results of the previous sections the flow rate in the trailing films is

$$
\begin{aligned}
Q_{R}^{ \pm}= & -C a^{5 / 3} T_{0, R}^{ \pm}-C a^{2}\left(T_{1, R}^{ \pm}-\frac{B o \cos (\alpha)\left(T_{0, R}^{ \pm}\right)^{3}}{3}\right) \\
& +O\left(C a^{7 / 3}\right) .
\end{aligned}
$$

Then the rate at which the plug volume $V$ changes with time is

$$
\frac{d V}{d t}=\left(Q_{R}^{+}+Q_{R}^{-}\right)-\left(Q_{F}^{+}+Q_{F}^{-}\right) .
$$

Figure 12(a) shows a plot of $d V / d t$ against $C a$ for a given $B o$ and precursor film thickness. When $C a$ is small, the trailing film is thin and the volume flux is dominated by gravitational drainage in the precursor films, which can cause the plug volume to increase $(d V / d t>0)$ for $\alpha>\pi / 2$ and decrease $(d V / d t<0)$ for $\alpha<\pi / 2$. As $C a$ increases, two opposing effects come into play: the plug picks up more fluid from the precursor films, but also deposits more liquid as the trailing films become thicker. Therefore, for a fixed value of the precursor film thickness, $d V / d t$ passes through a positive maximum and then becomes negative at a critical capillary number $C a_{\mathrm{c}}$ beyond which the plug volume always decreases and the plug eventually ruptures. Figure 12(b) shows $C a_{\mathrm{c}}$ as a function of $B o$ for different values of $\alpha$ and $h_{\infty, F}^{ \pm}$. When the trailing films become thinner with increasing $B o$ (see Fig. 4), $C a_{\mathrm{c}}$ increases, while the opposite is true for orientations at which the trailing films become thicker with increasing $B o$. $C a_{\mathrm{c}}$ also increases as $h_{\infty, F}^{ \pm}$increases. Thus plug rupture is promoted by higher propagation speeds and thinner precursor films, and is more likely to occur in airways in which the plug motion is opposed by gravity.

\section{Summary}

We have shown that gravity and channel orientation can have significant effects on the propagation of a liquid plug through a liquid-lined channel. Two different scaling regimes were identified at low capillary number. In the viscocapillary regime which exists for Bond numbers less than an orientation dependent critical value $B o_{\mathrm{c}}$, the trailing film thickness, length of the transition region, and flow contribution to the 

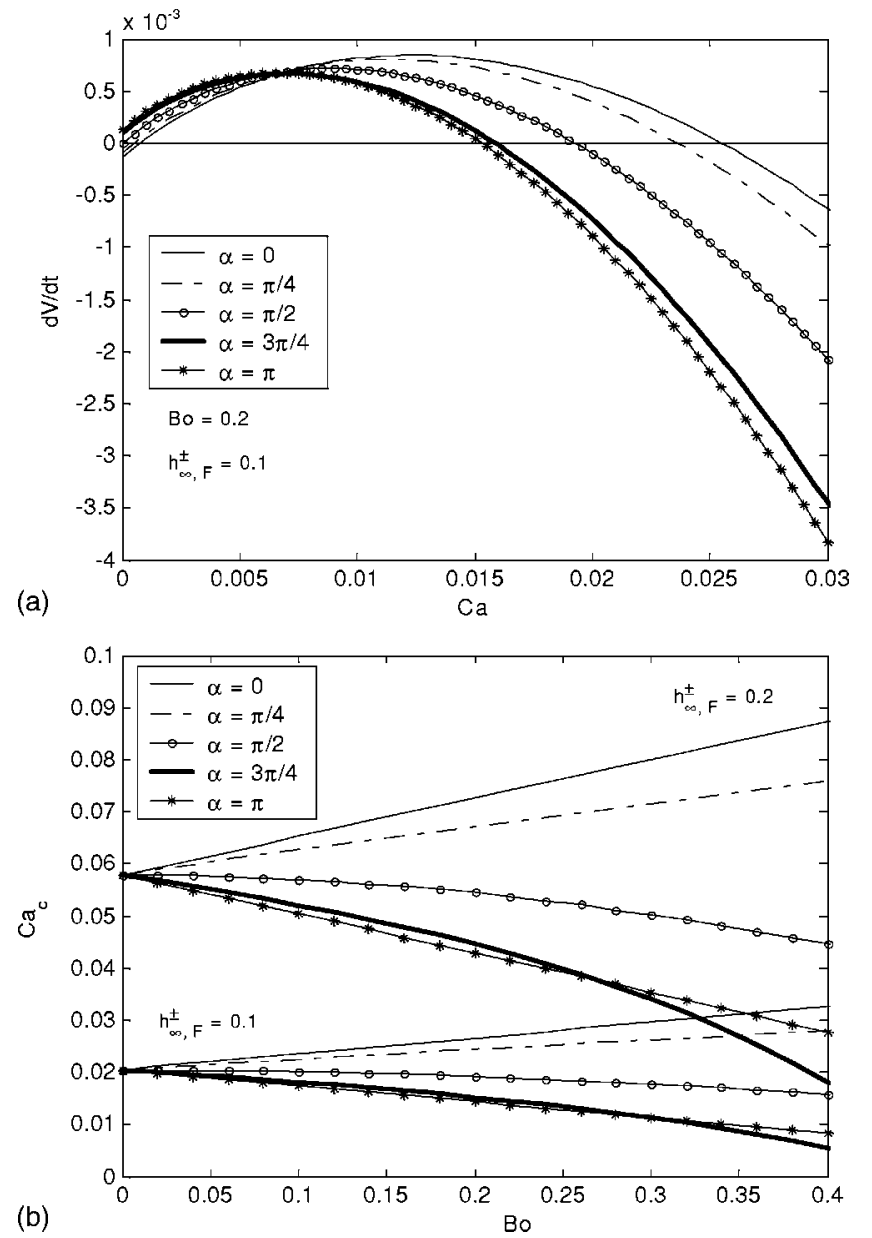

FIG. 12. (a) Rate of change of volume of the plug vs $\mathrm{Ca}$ and (b) critical capillary number $C a_{\mathrm{c}}$ vs $B o$ for eventual plug rupture.

pressure drop across the plug scale as $\mathrm{Ca}^{2 / 3}, \mathrm{Ca}^{1 / 3}$, and $\mathrm{Ca}^{2 / 3}$, respectively. The first higher-order correction to the film thickness occurs at $O(\mathrm{Ca})$ compared to $O\left(\mathrm{Ca}^{4 / 3}\right)$ in the nogravity case. A crossover to the gravitational regime occurs at $B o=B o_{c}$ where these quantities scale as $C a^{1 / 2}, C a^{1 / 6}$, and $C a^{1 / 2}$, respectively. For small increments of the Bond number over its critical value $\left(B o=B o_{c}+C a^{1 / 6} B\right)$ the scaling of the film thickness and transition region length remain unchanged, but the pressure drop scales as $\mathrm{Ca}^{1 / 6}$. For higher Bond numbers the small capillary number asymptotics break down and the full Stokes equations must be solved.

The results of the asymptotic analysis were used to examine the effect of gravity on two features of physiological interest, prebifurcation asymmetry in the liquid distribution in the channel and criteria for plug rupture. Asymmetry in the liquid distribution was quantified by calculating the ratio of liquid volumes above and below the centerline of the channel, $V_{R}$. $V_{R}$ depends strongly on $\alpha$ and decreases with $B o$, but increases with the plug length $L_{p}$. An interesting result was that $V_{R}$ decreases with $\mathrm{Ca}$, i.e., the asymmetry in the liquid distribution increases with the plug propagation speed. Thus higher propagation speeds may promote unequal plug splitting at airway bifurcations and increase the inhomogeneity in the overall liquid distribution in the lung. This result is valid in small airways at low speeds when liquid inertia is negligible, and is in contrast to the behavior expected in large airways in which higher speeds would increase inertial effects and result in a more uniform liquid distribution.

Criteria for plug rupture were obtained by determining a critical capillary number $C a_{c}$ as a function of $B o, \alpha$ and the precursor film thickness, above which the plug eventually ruptures. Plug rupture limits the depth of penetration of instilled liquid into the lung and thus affects the overall delivery. We found that, in general, plug rupture occurs at lower speeds (lower $\mathrm{Ca}_{c}$ ) when the plug motion is opposed by gravity and in channels with thinner precursor films.

These calculations are based on a two-dimensional (2D) channel geometry, whereas a real airway has a cylindrical geometry in which the shape of the interface is not symmetric about the airway axis and the trailing film thickness in a cross-section normal to the axis varies with the azimuthal angle. Analogously, in the 2D channel, gravitational effects distort the interface shape and lead to different film thickness on the upper and lower walls. Many of the interesting results of our study, such as the nonhomogeneous liquid distribution and the $(B o, \alpha)$ dependence of critical capillary number for plug rupture are a result of this gravitationally induced asymmetry and therefore will be present in cylindrical airways as well. While the quantitative dependence of the results on the system parameters will depend on the geometry, the scaling relations in the limit of small $\mathrm{Ca}$ are identical in either case and therefore the results for the 2D channel will provide insight into the behavior in the cylindrical geometry as well.

The asymptotic analysis presented here provides insight into the physical processes influencing the low capillary number dynamics of plugs in small airways with plug lengths comparable to the airway radius, and does not address the transport of instilled liquid from large airways to smaller ones. This initial transport process is important since it determines input parameters to the current model such as the plug length, which affects liquid distribution and plug rupture. Since inertial effects become important at the length and velocity scales prevalent in large airways, the equations of motion need to be solved numerically. Recent studies have examined finite Reynolds number effects in gravity-free Bretherton type problems propagation using scaling arguments ${ }^{49,50}$ and numerical computations. ${ }^{51-54}$ These studies, in general, have found that inertial effects lead to a slight decrease in the trailing film thickness at Reynolds numbers less than about 100; however, there is a large increase in the driving pressure and significant changes in the flow and pressure fields in the liquid. ${ }^{53,54}$

\section{ACKNOWLEDGMENTS}

This work was supported by NIH Grants No. HL41126, No. HL64373, NSF Grant No. BES-9820967, and NASA Grant No. NAG3-2196.

\section{APPENDIX}

An arc-length coordinate system $(s, \theta(s))$ is defined where $s$ is the arc length along the air-liquid interface. The origin is located at the contact point of the static interface 
with the upper wall. The interface transforms as $\left(x, h^{ \pm}(x)\right) \mapsto(\zeta(s), \eta(s))$ with

$$
\begin{aligned}
& \frac{d \zeta}{d s}= \begin{cases}\cos (\theta) & \text { (rear) } \\
-\cos (\theta) & \text { (front) },\end{cases} \\
& \frac{d \eta}{d s}=-\sin (\theta) .
\end{aligned}
$$

The curvature is given by $\pm h_{x x}^{ \pm} /\left(1+h_{x}^{ \pm^{2}}\right)^{3 / 2}=-d \theta / d s$ and the normal stress balance without the viscous term is

$$
\frac{d \theta}{d s}=\delta p-B o \zeta(s) \cos (\alpha)+B o \eta(s) \sin (\alpha) .
$$

$\zeta(s), \eta(s), \theta(s)$, and $\delta p$ are expanded in the perturbation series (15) to obtain equations in the statics region at each order.

\section{Equations at $O\left(\mathrm{Ca}^{0}\right)$}

The equations at leading order are identical in the viscocapillary and gravitational regimes,

$$
\begin{aligned}
& \frac{d \zeta_{0}}{d s}= \begin{cases}\cos \left(\theta_{0}(s)\right) & (\text { rear }) \\
-\cos \left(\theta_{0}(s)\right) & \text { (front) })\end{cases} \\
& \frac{d \eta_{0}}{d s}=\sin \left(\theta_{0}(s)\right), \\
& \frac{d \theta_{0}}{d s}=\Delta p_{0}-B o \zeta_{0}(s) \cos (\alpha)+B o \eta_{0}(s) \sin (\alpha) .
\end{aligned}
$$

Boundary conditions (20), (21), (40), (41), and (42a) become

$$
\zeta_{0}=0, L_{P} ; \eta_{0}=1 ; \theta_{0}=0, \quad s=0,
$$

$$
\left.\begin{array}{l}
\zeta_{2 / 3}=0 \\
\left.\eta_{2}=-\left(A_{0}^{+} X_{0}^{+2} / 2 T_{0}^{+}+A_{1}^{+} X_{0}^{+}+A_{2}^{+} T_{0}^{+}\right)\right\}, \quad s=0, \\
\eta_{3}=0 \\
\zeta_{2 / 3}=0 \\
\eta_{2}=\left(A_{0}^{-} X_{0}^{-2} / 2 T_{0}^{-}+A_{1}^{-} X_{0}^{-}+A_{2}^{-} T_{0}^{-}\right) \\
\eta_{3}=\left(-\frac{B o_{\mathrm{c}} \cos (\alpha) X_{0}^{-3}}{6}+\frac{A_{0}^{-} X_{0}^{-2}}{2 T_{0}}+A_{1}^{-} X_{0}^{-}+A_{2}^{-} T_{0}^{-}\right)
\end{array}\right\}, \quad s=s_{\pi} .
$$

The boundary conditions follow from (22), (30) and (51), (52):

$$
\begin{aligned}
& \frac{d \zeta_{2 / 3}}{d s}= \begin{cases}-\sin \left(\theta_{0}\right) \theta_{2 / 3} & \text { (rear) } \\
\sin \left(\theta_{0}\right) \theta_{2 / 3} & \text { (front) },\end{cases} \\
& \frac{d \eta_{2 / 3}}{d s}=-\cos \left(\theta_{0}\right) \theta_{2 / 3}, \\
& \frac{d \theta_{2 / 3}}{d s}=\Delta p_{2 / 3}-B o \zeta_{2 / 3} \cos (\alpha)+B o \eta_{2 / 3} \sin (\alpha) .
\end{aligned}
$$

which provide three conditions to integrate (A4)-(A6) and two more to determine $\Delta p_{0}$ and $s_{\pi}$. The smoothness condition (22) implies that

$$
\theta_{0}=\pi / 2, \quad \theta_{i}=0(i>0), \quad \zeta_{0}=x_{t}, \quad s=s_{\pi / 2} .
$$

The condition (42b) used to determine $B o_{\mathrm{c}}$ in the gravitational regime becomes

$$
\frac{d \theta_{0}}{d s}=0, s=s_{\pi} .
$$

\section{Equations at $O\left(\mathrm{Ca}^{1 / 2}\right)$ and $O\left(\mathrm{Ca}^{2 / 3}\right)$}

$\left(\zeta_{2}(s), \eta_{2}(s), \theta_{2}(s), \delta p_{2}\right)$ at $O\left(C a^{2 / 3}\right)$ in the viscocapillary regime and $\left(\zeta_{3}(s), \eta_{3}(s), \theta_{3}(s), \delta p_{3}\right)$ at $O\left(C a^{1 / 2}\right)$ in the gravitational regime, which satisfy the same equations
When $\alpha=0$ or $\pi$, solutions are symmetric about $y=0$, i.e., $s=s_{\pi / 2}$. The domain of integration is $\left(s_{\pi / 2}, s_{\pi}\right)$ and the conditions at $s=0$ are replaced by

$$
\begin{aligned}
& \eta_{2 / 3}=0, \quad s=s_{\pi / 2}, \\
& \zeta_{2 / 3}=0, \quad s=s_{\pi} .
\end{aligned}
$$

${ }^{1}$ L. K. Hastings, W. H. Renfro, and R. Sharma, "Comparison of beractant and calfactant in a neonatal intensive care unit," Am. J. Health Syst. Pharm. 61, 257 (2004).

${ }^{2}$ A. H. Jobe, "Pulmonary surfactant therapy," N. Engl. J. Med. 328, 861 (1993).

${ }^{3}$ H. Yapicioglu, D. Yildizdas, I. Bayram, Y. Sertdemir, and H. L. Yilmaz, "The use of surfactant in children with acute respiratory distress syndrome: Efficacy in terms of oxygenation, ventilation, and mortality," Pulm. Pharmacol. Ther. 16, 327 (2003).

${ }^{4}$ B. P. Fuhrman, P. R. Paczan, and M. DeFrancisis, "Perfluorocarbon- 
associated gas exchange," Crit. Care Med. 19, 712 (1991).

${ }^{5}$ R. B. Hirschl, M. Croce, D. Gore, H. Wiedemann, K. Davis, J. Zwischenberger, and R. H. Bartlett, "Prospective, randomized, controlled pilot study of partial liquid ventilation in adult acute respiratory distress syndrome," Am. J. Respir. Crit. Care Med. 165, 781 (2002).

${ }^{6}$ P. N. Cox, H. Frndova, O. Karlsson, S. Holowka, and C. A. Bryan, "Fluorocarbons facilitate lung recruitment," Intensive Care Med. 29, 2297 (2003).

${ }^{7}$ K. Mikawa, K. Nishina, Y. Takao, and H. Obara, "Efficacy of partial liquid ventilation in improving acute lung injury induced by intratracheal acidified infant formula: Determination of optimal dose and positive endexpiratory pressure level," Crit. Care Med. 32, 209 (2004).

${ }^{8}$ D. J. Weiss, T. P. Strandjord, J. C. Jackson, J. G. Clark, and D. Liggitt, "Perfluorochemical liquid-enhanced adenoviral vector distribution and expression in lungs of spontaneously breathing rodents," Exp. Lung Res. 25, 317 (1999)

${ }^{9}$ D. J. Smith, L. M. Gambone, T. Tarara, D. R. Meays, L. A. Dellamary, C. M. Woods, and J. Weers, "Liquid dose pulmonary instillation of gentamicin PulmoSpheres (R) formulations: Tissue distribution and pharmacokinetics in rabbits," Pharm. Res. 18, 1556 (2001).

${ }^{10}$ J. J. Haitsma, U. Lachmann, and B. Lachmann, "Exogenous surfactant as a drug delivery agent," Adv. Drug Delivery Rev. 47, 197 (2001).

${ }^{11}$ Y. L. Zhang, O. K. Matar, and R. V. Craster, "A theoretical study of chemical delivery within the lung using exogenous surfactant," Med. Eng. Phys. 25, 115 (2003).

${ }^{12}$ S. Iqbal, S. Ritson, I. Prince, J. Denyer, and M. L. Everard, "Drug delivery and adherence in young children," Pediatr. Pulmonol 37, 311, (2004).

${ }^{13}$ P. B. Myrdal, K. L. Karlage, S. W. Stein, B. A. Brown, and A. Haynes, "Optimized dose delivery of the peptide cyclosporine using hydrofluoroalkane-based metered dose inhalers," J. Pharm. Sci. 93, 1054 (2004).

${ }^{14}$ F. F. Espinosa and R. D. Kamm, "Meniscus formation during tracheal instillation of surfactant," J. Appl. Physiol. 85, 266 (1998).

${ }^{15}$ K. J. Cassidy, J. L. Bull, M. R. Glucksberg, C. A. Dawson, S. T. Haworth, R. B. Hirschl, N. Gavriely, and J. B. Grotberg, "A rat lung model of instilled liquid transport in the pulmonary airways," J. Appl. Physiol. 90, 1955 (2001).

${ }^{16}$ J. C. Anderson, R. C. Molthen, C. A. Dawson, S. T. Haworth, J. L. Bull, M. R. Glucksberg, and J. B. Grotberg, "Effect of ventilation rate on instilled surfactant distribution in the pulmonary airways of rats," J. Appl. Physiol. 97, 45 (2004)

${ }^{17}$ J. L. Bull, S. Tredici, E. Komori, D. O. Brant, J. B. Grotberg, and R. B. Hirschl, "Distribution dynamics of perfluorocarbon delivery to the lungs: An intact rabbit model," J. Appl. Physiol. 96, 1633 (2004).

${ }^{18}$ K. J. Cassidy, N. Gavriely, and J. B. Grotberg, "Liquid plug flow in straight and bifurcating tubes," J. Biomech. Eng. 123, 580 (2001).

${ }^{19}$ V. Sauret, R. M. Halson, I. W. Brown, J. S. Fleming, and A. G. Bailey, "Study of the three-dimensional geometry of the central conducting airways in man using computed tomographic (CT) images," J. Anat. 200, 123 (2002)

${ }^{20}$ F. P. Bretherton, "The motion of long bubbles in tubes," J. Fluid Mech. 10, 166 (1961).

${ }^{21}$ C.-W. Park and G. M. Homsy, "Two-phase displacement in Hele Shaw cells: theory," J. Fluid Mech. 139, 291 (1984).

${ }^{22} \mathrm{H}$. Wong, C. J. Radke, and S. Morris, "The motion of long bubbles in polygonal capillaries. Part 1. Thin films," J. Fluid Mech. 292, 71 (1995).

${ }^{23} \mathrm{H}$. Wong, C. J. Radke, and S. Morris, "The motion of long bubbles in polygonal capillaries. Part 2. Drag, fluid pressure and fluid flow," J. Fluid Mech. 292, 95 (1995).

${ }^{24}$ L. W. Schwartz, H. M. Princen, and A. D. Kiss, "On the motion of bubbles in capillary tubes," J. Fluid Mech. 172, 259 (1986).

${ }^{25}$ G. M. Ginley and C. J. Radke, "Influence of soluble surfactants on the flow of long bubbles through a cylindrical capillary," ACS Symp. Ser. 396, 480 (1988).

${ }^{26}$ J. Ratulowski and H.-C. Chang, "Marangoni effects of trace impurities on the motion of long gas bubbles in capillaries," J. Fluid Mech. 210, 303 (1990).
${ }^{27} \mathrm{C}$.-W. Park, "Influence of soluble surfactants on the motion of finite bubble in a capillary tube," Phys. Fluids A 4, 2335 (1992).

${ }^{28}$ K. J. Stebe and D. Bathés-Biesel, "Marangoni effects of adsorptiondesorption controlled surfactants on the leading end of an infinitely long bubble in a capillary," J. Fluid Mech. 286, 25 (1995).

${ }^{29}$ A. Mazouchi and G. M. Homsy, "Thermocapillary migration of long bubbles in cylindrical capillary tubes," Phys. Fluids 12, 542 (2000).

${ }^{30}$ A. Mazouchi and G. M. Homsy, "Thermocapillary migration of long bubbles in polygonal tubes. I. Theory," Phys. Fluids 13, 1594 (2001).

${ }^{31} \mathrm{D}$. Halpern and O. E. Jensen, "A semi-infinite bubble advancing into a planar tapered channel," Phys. Fluids 14, 431 (2002).

${ }^{32}$ P. D. Howell, S. L. Waters, and J. B. Grotberg, "The propagation of a liquid bolus along a liquid-lined flexible tube,” J. Fluid Mech. 406, 309 (2000).

${ }^{33}$ O. E. Jensen, M. K. Horsburgh, D. Halpern, and D. P. Gaver, "The steady propagation of a bubble in a flexible-walled channel: Asymptotic and computational models," Phys. Fluids 14, 443 (2002).

${ }^{34} \mathrm{~S}$. L. Waters and J. B. Grotberg, "The propagation of a surfactant laden liquid plug in a capillary tube," Phys. Fluids 14, 471 (2002).

${ }^{35}$ M. H. Jensen, A. Libchaber, P. Pelce, and G. Zocchi, "Effect of gravity on the Saffman-Taylor meniscus: Theory and experiment," Phys. Rev. A 35, 2221 (1987)

${ }^{36} \mathrm{D}$. Lasseux and M. Quintard, "Film thickness behind a receding meniscus," C. R. Acad. Sci., Ser. II: Mec., Phys., Chim., Sci. Terre Univers 313, 1375 (1991).

${ }^{37}$ D. Lasseux, "Drainage in a capillary-A complete approximated description of the interface," C. R. Acad. Sci., Ser. IIb: Mec., Phys., Chim., Astron. 321, 125 (1995).

${ }^{38}$ D. Quéré, "Fluid coating on a fiber," Annu. Rev. Fluid Mech. 31, 347 (1999).

${ }^{39}$ L. D. Landau and V. G. Levich, "Dragging of a liquid by a moving plate," Acta Physicochim. URSS 17, 42 (1942).

${ }^{40} \mathrm{~B}$. V. Derjaguin, "On the thickness of the liquid film adhering to the walls of a vessel after emptying," Acta Physicochim. URSS 20, 349 (1943).

${ }^{41}$ S. D. R. Wilson, "The drag-out problem in film coating theory," J. Eng. Math. 16, 209 (1982).

${ }^{42}$ B. V. Derjaguin and S. M. Levi, Film Coating Theory (Focal, London, 1964).

${ }^{43}$ A. de Ryck and D. Quere, "Gravity and inertia effects in plate coating," J. Colloid Interface Sci. 203, 278 (1998).

${ }^{44}$ D. A. White and J. A. Tallmadge, "Theory of drag out of liquids on flat plates," Chem. Eng. Sci. 20, 33 (1965).

${ }^{45}$ J. R. Cash, G. Moore, and R. W. Wright, "An automatic continuation strategy for the solution of singularly perturbed linear 2-point boundaryvalue-problems," J. Comput. Phys. 122, 266 (1995).

${ }^{46}$ J. R. Cash, G. Moore, and R. W. Wright, "An automatic continuation strategy for the solution of singularly perturbed nonlinear boundary value problems," ACM Trans. Math. Softw. 27, 245 (2001).

${ }^{47}$ D. M. King, Z. D. Wang, H. J. Palmer, B. A. Holm, and R. H. Notter, "Bulk shear viscosities of endogenous and exogenous lung surfactants," Am. J. Physiol. 282, L277 (2002).

${ }^{48}$ M. Van Dyke, Perturbation Methods in Fluid Mechanics (Academic, Boston, 1964).

${ }^{49} \mathrm{P}$. Aussillous and D. Quere, "Quick deposition of a fluid on the wall of a tube," Phys. Fluids 12, 2367 (2000).

${ }^{50}$ A. de Ryck, "The effect of weak inertia on the emptying of a tube," Phys. Fluids 14, 2102 (2002).

${ }^{51}$ M. D. Giavedoni and F. A. Saita, "The axisymmetric and plane cases of a gas phase steadily displacing a Newtonian liquid-A simultaneous solution of the governing equations," Phys. Fluids 9, 2420 (1997).

${ }^{52}$ M. D. Giavedoni and F. A. Saita, "The rear meniscus of a long bubble steadily displacing a Newtonian liquid in a capillary tube," Phys. Fluids 11, 786 (1999).

${ }^{53}$ M. Heil, "Finite Reynolds number effects in the Bretherton problem," Phys. Fluids 13, 2517 (2001).

${ }^{54}$ H. Fujioka and J. B. Grotberg, "Steady propagation of a liquid plug in a two-dimensional channel," J. Biomech. Eng. 126, 567 (2004). 